\title{
Advanced Packaging Technology for High Frequency Photonic Applications
}

Marcelino G. Armendariz, G. Ronald Hadley, Mial E. Warren, Robert E. Smith, Stan H. Kravitz, Joel R. Wendt, Tom M. Bauer, Rick Corless, James C. Word, Pam K. Seigal

Prepared by

Sandia National Laboratories

Albuquerque, New Mexico 87185 and Livermore, California 94550

for the United States Department of Energy

under Contract DE-AC04-94AL85096.

Approved for public release; distribution is unlimited.

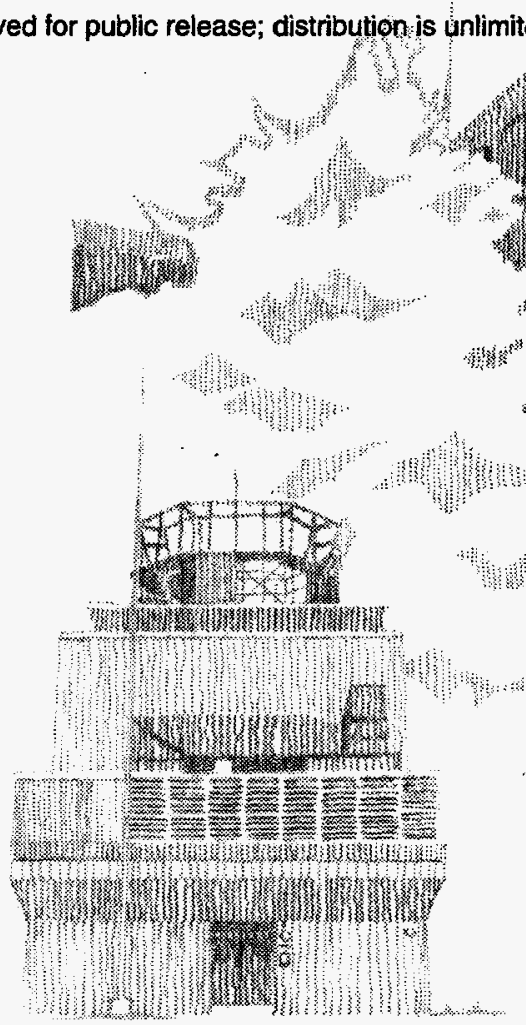

SF $29000(8-81)$

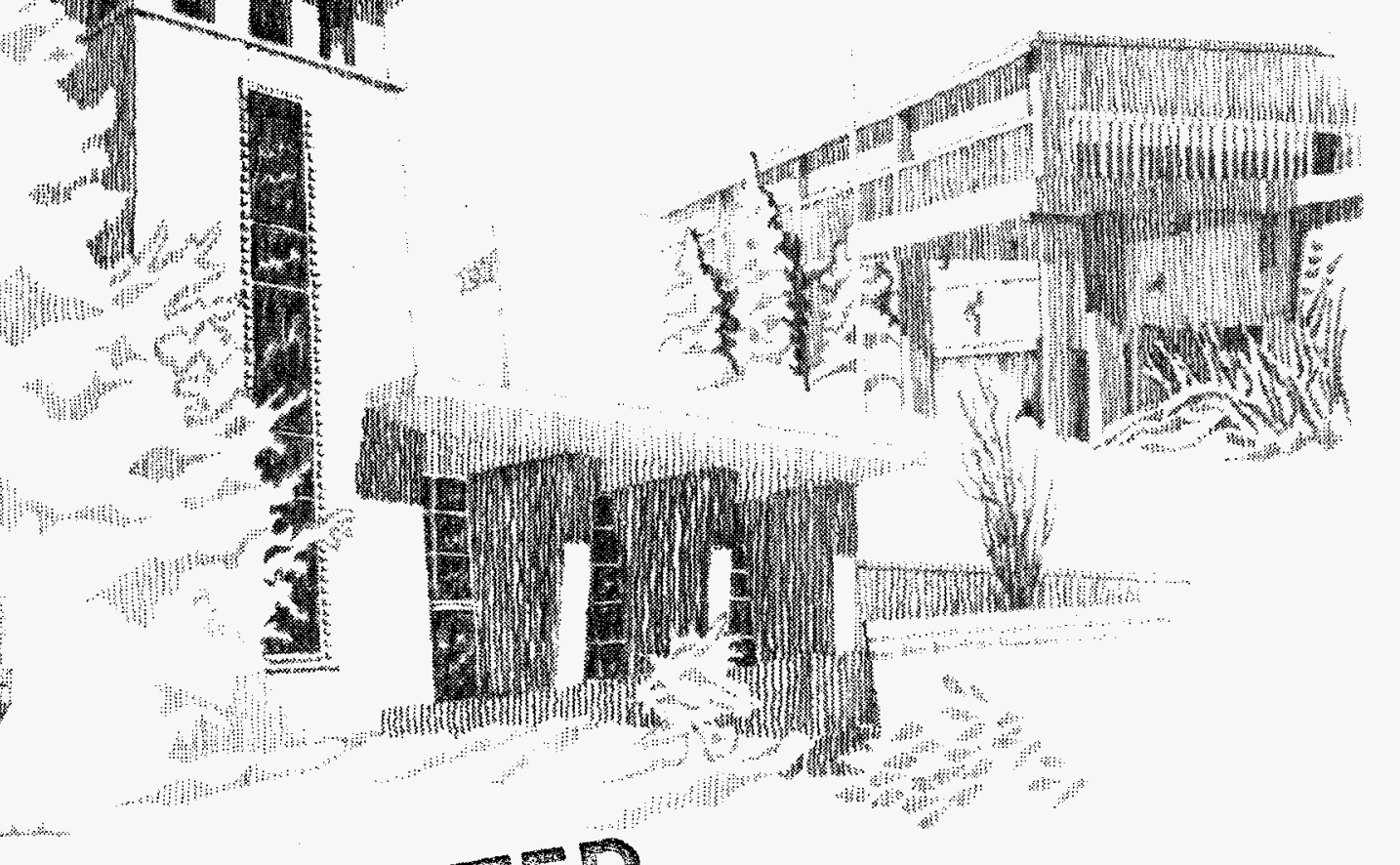

96.

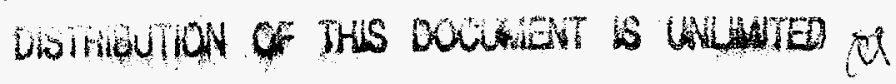


Issued by Sandia National Laboratories, operated for the United States Department of Energy by Sandia Corporation.

NOTICE: This report was prepared as an account of work sponsored by an agency of the United States Government. Neither the United States Government nor any agency thereof, nor any of their employees, nor any of their contractors, subcontractors, or their employees, makes any warranty, express or implied, or assumes any legal liability or responsibility for the accuracy, completeness, or usefulness of any information, apparatus, product, or process disclosed, or represents that its use would not infringe privately owned rights. Reference herein to any specific commercial product, process, or service by trade name, trademark, manufacturer, or otherwise, does not necessarily constitute or imply its endorsement, recommendation, or favoring by the United States Government, any agency thereof or any of their contractors or subcontractors. The views and opinions expressed herein do not necessarily state or reflect those of the United States Government, any agency thereof or any of their contractors.

Printed in the United States of America. This report has been reproduced directly from the best available copy.

Available to DOE and DOE contractors from Office of Scientific and Technical Information

PO Box 62

Oak Ridge, TN 37831

Prices available from (615) 576-8401, FTS 626-8401

Available to the public from

National Technical Information Service

US Department of Commerce

5285 Port Royal Rd

Springfield, VA 22161

NTIS price codes

Printed copy: A03

Microfiche copy: A01 


\section{DISCLAIMER}

Portions of this document may be illegible in electronic image products. Images are produced from the best available original document. 
SAND96-0658

Unlimited Release

Printed March 1996

\title{
Advanced Packaging Technology for High Frequency Photonic Applications
}

\author{
Marcelino G. Armendariz \\ Advanced Devices \& Applications Department \\ G. Ronald Hadley and Mial E. Warren \\ Photonics Research Department \\ Robert. E. Smith \\ Semiconductor Materials Department \\ Stan H. Kravitz and Joel R. Wendt \\ Compound Semiconductor Technology Department \\ Tom M. Bauer, Rick Corless, and James C. Word \\ Microelectronics Facility Operations Team Department \\ Pam K. Seigal \\ Advanced Packaging Department \\ Sandia National Laboratories \\ Albuquerque, New Mexico 87185-0603
}

\begin{abstract}
An advanced packaging concept has been developed for optical devices. This concept allows multiple fibers to be coupled to photonic integrated circuits, with no fiber penetration of the package walls. The principles used to accomplish this concept involves a second-order grating to couple light in or out of the photonic circuit, and a binary optic lens which receives this light and focuses it into a single-mode optical fiber. Design, fabrication and electrical/optical measurements of this packaging concept are described.
\end{abstract}




\section{Figures}

Fig. 1. Package Concept

Fig. 2. Design parameters for the basic waveguide, together with the fundamental mode profile.

Fig. 3. Linearly flared waveguide geometry.

Fig. 4. Near-field intensity profile (to scale) of the input beam (Fig. 2) after propagating $1000 \mathrm{~mm}$ down the flared waveguide.

Fig. 5. Far-field pattern of the expanded beam whose near-field profile is shown in Fig. 4.

Fig. 6. Geometry used for model calculations of grating emission (not to scale).

Fig. 7. Predicted far-field emission from grating into air.

Fig. 8. Test structure for development of advanced packaging technology

Fig. 9. Vertical Gratings with Bragg Reflectors

Fig. 10. Far-field measurements of the beam exiting the grating.

Fig. 11. 45 degrees blazed gratings.

Fig. 12. A scanning electron micrograph of a silicon 4 phase-level binary optic lens.

Fig. 13. Anamorphic binary optic lens

Fig. 14. Insertion loss and return loss of photonic package with PIC.

Fig. 15. S21dB of PIC loss and S21dB of PIC launched to coplanar transition and $\mathrm{V}$-connectors.

Fig. 16. Calculation of width $\mathrm{W}$, and distance $\mathrm{D}$ for $62.5 \mu \mathrm{m}$ fiber.

Fig. 17. SEM of single-mode fiber in a silicon V-groove.

Fig. 18. SEM of laser drilled hole on a silicon substrate on an etched window.

Fig. 19. Complete mechanical assembly of packaged APPA concept.

Fig. 20. Configuration for coupling from ridge waveguide to single-mode fiber via waveguide grating-coupler and silicon microlens.

Fig. 21. On-wafer optical fiber probe and if probe test station. 


\section{Acknowledgment}

The authors would like to thank W. Doyle Miller for his laser drilling support and Charles E. Sandoval for the microwave characterization. 


\section{Contents}

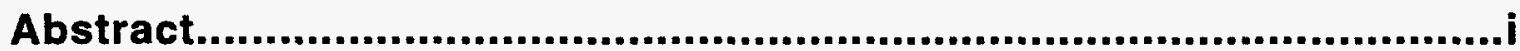

Acknowledgment....................................................................ii

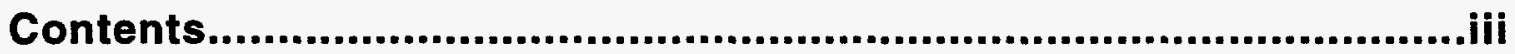

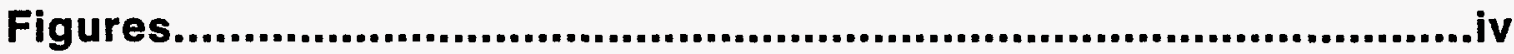

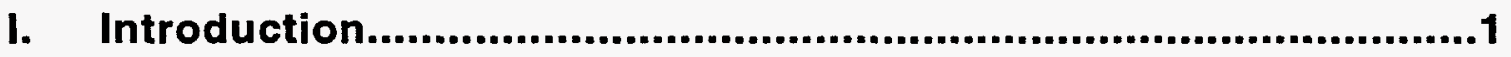

II. Concept of Photonic Package......................................................1

III. Design of waveguide, adiabatic expander and surface-emitting grating...........................................................................................3

A. Input Waveguide....................................................................3

B. Adiabatic Waveguide Flare...........................................................3

C. Grating Design....................................................................6

IV. Fabrication of Grating Couplers...............................................8

V. Binary Optic Lens Design and Fabrication...............................10

VI. Microwave Launch to Photonic Device.....................................12

VII. Packaging Assembly.........................................................13

A. V-Grooves in silicon.............................................................13

B. Laser Drilling of Vertical Vias in Silicon.......................................15

C. Assembly...........................................................................16

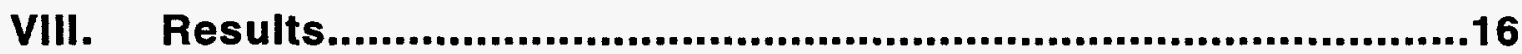

IX. Conclusions.......................................................................18

References...............................................................................19

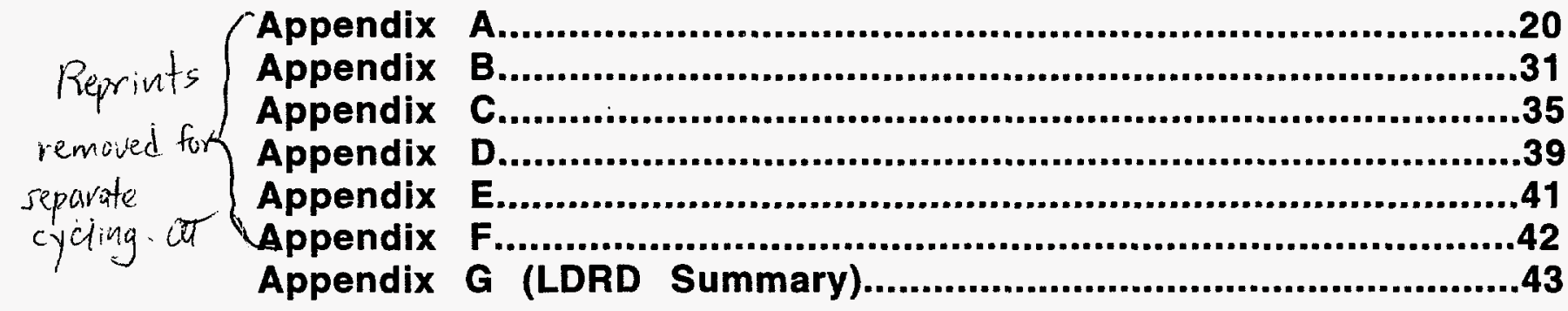




\section{Introduction}

One of the primary challenges in the development and application of complex photonic integrated circuits (PICs) is the packaging of the devices. The coupling of single mode fibers to a semiconductor guided-wave device is an expensive manufacturing step and a major part of the system losses. For complex circuits in which many channels need to be coupled off of the chip, the difficulties are enormous and far from solved. The requirements for a successful packaging technology for multiple channel PICs include low loss coupling from single-mode, rectangular, semiconductor waveguides to single-mode fibers, hermetic sealing of the package against environmental conditions, a means of bringing a large number of optical signal channels out of the chip without greatly increasing its size and an efficient means of aligning each of a large number of single-mode fibers with minimum losses.

The demand for high frequency PICs has allowed us to develop photonic waveguide devices coupled to single-mode fiber and packaging techniques for applications at $1.3 \mu \mathrm{m}$ wavelength. The $1.3 \mu \mathrm{m}$ wavelength region is important because it is the basis for the vast majority of the installed fiber cable in this country. At this wavelength, lightwave signal dispersion vanishes and high power high-coherence diode-pumped Nd:YAG lasers are available.

At present there is a lack of techniques for efficient coupling of high-frequency electrical and optical signals to photonic integrated circuits. This represents a gap in the Sandia National Laboratories microelectronic/photonic capability, since for current applications, devices and packages require larger numbers of high-speed electrical and optical input/output channels and higher levels of integration to achieve state-of-the-art performance. Most device applications presently being pursued within the microelectronics and photonics core competency require precision packaging and integration to be realized as useful prototypes. This project will address advanced packaging using high frequency photonic waveguide devices coupled to optical fibers.

The focus of activities will be the establishment of technologies at Sandia in the Compound Semiconductor Research Laboratory (CSRL). The proposed devices were designed, grown, fabricated, and characterized to help us understand the difficulties associated with optical-fiber-coupled photonic devices.

\section{Concept of Photonic Package}

Historically, obtaining efficient coupling from single-mode waveguides in high performance GaAs modulator devices to single-mode fiber has been difficult. The reasons are; (1) the large modal mismatch between the elliptical waveguide output and the gaussian profile of the optical fiber; and (2) the large 
NA difference ( 0.9 for the waveguide in one direction) and 0.16 for fiber. Despite this difficulty, there exists a need for packaging devices with multiple fiber outputs, that have been gang-aligned, efficiently coupled, and hermetically sealed. (The latter item will be very important in automotive or aerospace applications.) Instead of trying to have the fiber penetrate the package wall, the SNL approach to efficient coupling and hermeticity has been to allow light to penetrate the package wall. This has been accomplished by sending out the light normal to the waveguides and collecting it with a binary optic that focuses it on to a fiber outside the package. (Fig. 1) A basic test structure has been designed for prototype testing of photonic packaging technology that incorporates waveguide segments with phase modulators and second-order grating couplers as shown in Fig. 2. The photonic device along with high frequency (up to $50 \mathrm{GHz}$ ) electrical control is the basis for this LDRD and will be labeled as advanced photonic package assembly (APPA).

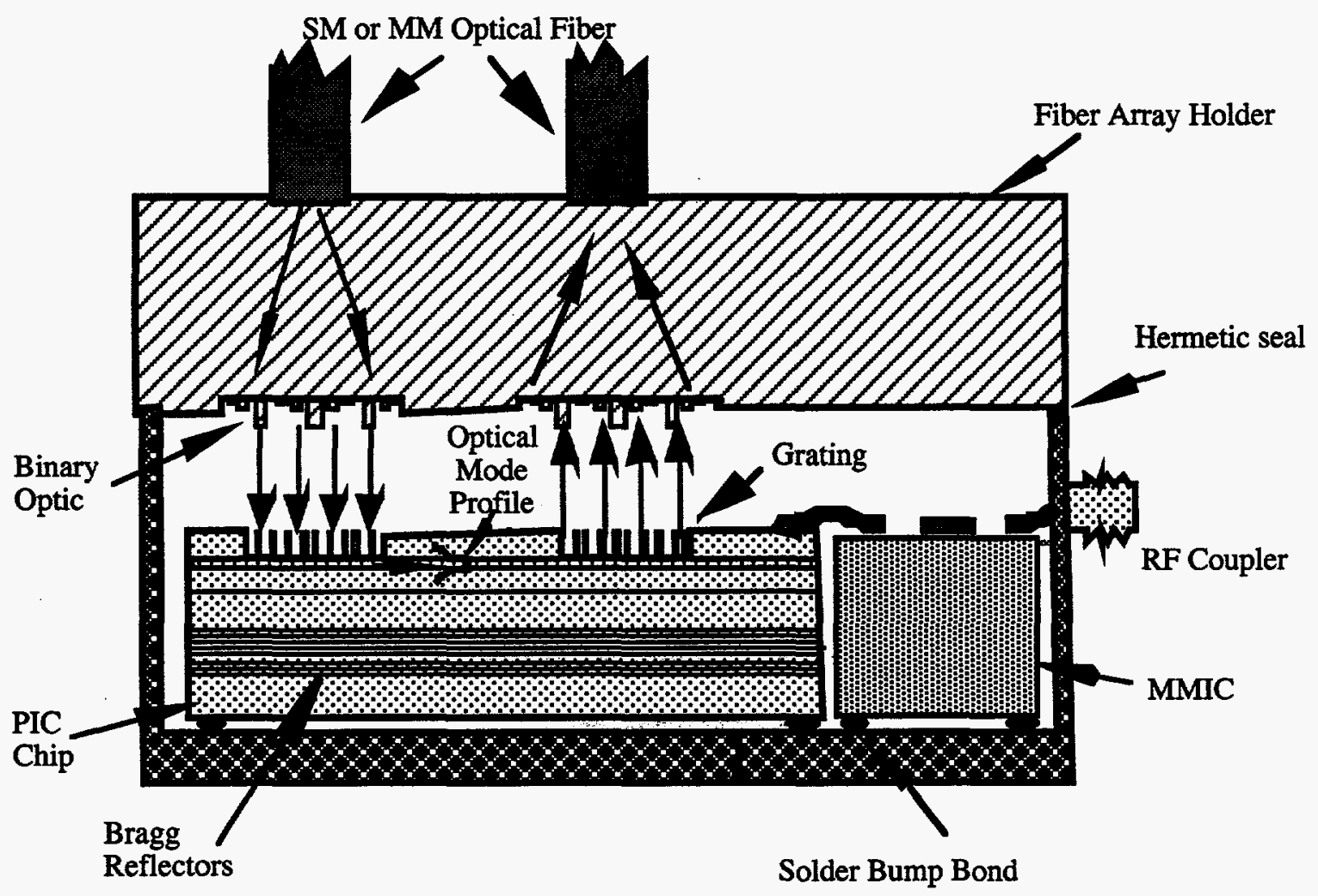

Fig. 1. Package Concept 


\section{Design of waveguide, adiabatic expander and surface-emitting grating}

\section{A. Input Waveguide}

The input waveguide has been kept as close to the standard COMPASS design as possible, owing to the close connection between the two applications.[1] However, in order to maximize the grating coupling efficiency, the rib height has been decreased so as to place the upper rib surface closer to the guided wave. The rib etch depth has been set to provide good confinement while still cutting off the second order mode. The resulting design is shown, together with the fundamental mode profile, in Fig. 2.

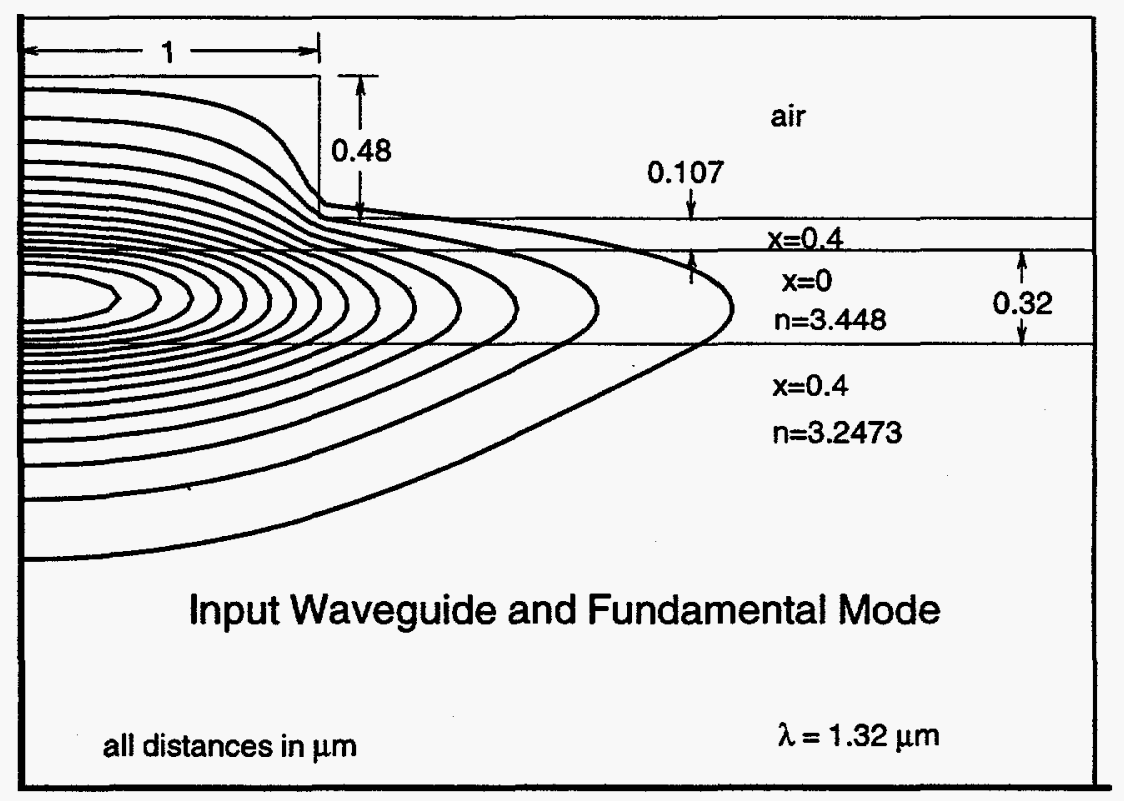

Fig. 2 Design parameters for the basic waveguide, together with the fundamental mode profile.

\section{B. Adiabatic Waveguide Flare}

The requirement of good beam quality for the output beam implies that aspect ratios be as close to unity as possible and the beam have low divergence. Both of these qualities are expected to aid in obtaining good coupling efficiency into the fiber. Since the input waveguide has only a $2 \mu \mathrm{m}$ width and coupling lengths for the grating are expected to be at least $100 \mu \mathrm{m}$, it follows that initially the beam is characterized by a very large aspect ratio. The small rib width also implies beam divergence in the plane of the wafer of \pm 5 deg that will become 
$\pm 20 \mathrm{deg}$ in air. Both these properties if left unchanged would make the beam difficult to focus.

Consequently, we have investigated the use of an adiabatic waveguide flare in order to decrease both the high aspect ratio and the lateral beam divergence and thus more closely match the small longitudinal divergence and large beam size that are expected to result from the grating outcoupler. After trying flares with a variety of shapes, we have concluded that a simple linear taper represents the optimum shape. The flare angle is directly related to the maximum output beam divergence desired, since Fourier components moving at smaller angles will not experience a reflection from the sidewalls. Thus, the output beam divergence of a flared waveguide decreases monotonically with the flare angle. However, small flare angles result in long taper lengths in order to expand the beam significantly. We have affected a compromise with the geometry shown in Fig. 3 by limiting the length to $1000 \mu \mathrm{m}$ while reducing the lateral divergence to a FWHM of $0.8 \mathrm{deg}$ (in the AIGaAs, corresponding to about 2.5 deg in air).

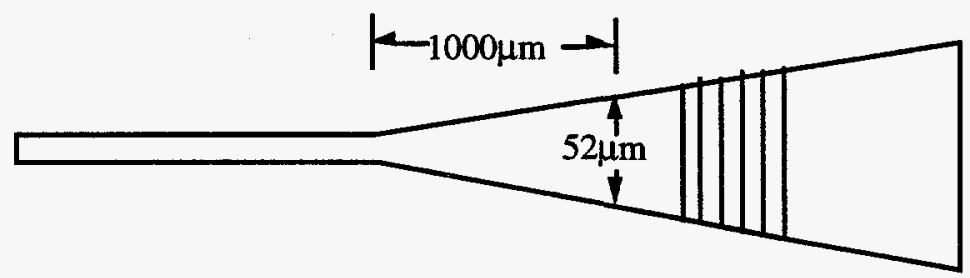

Fig. 3 Linearly flared waveguide geometry. The flare half-angle of $1.43 \mathrm{deg}$ results in a much gentler taper than shown in the Fig.. The grating (of length 200 $\mu \mathrm{m}$ ) is written starting $1000 \mu \mathrm{m}$ after the start of the flared section.

The effect of the flared waveguide section was modeled using 3-D wide-angle beam propagation starting with the waveguide eigenmode shown in Fig. 2 . The structure used in the calculation was that shown in Fig. 2, except that the rib width was allowed to increase linearly from $2 \mu \mathrm{m}$ to $52 \mu \mathrm{m}$ over a length of 1000 $\mu \mathrm{m}$. The resulting near- and far-field intensity profiles at the end of the flared section are shown in Fig.s 4 and 5. As is shown in Fig. 4, the beam waist has increased to about $38 \mu \mathrm{m}$ and is at this point expanding more slowly than the waveguide rib. Any attempt to straighten out the waveguide at this point (remove the taper) will result in unwanted sidewall reflections; consequently the grating should be written directly onto the tapered section. 


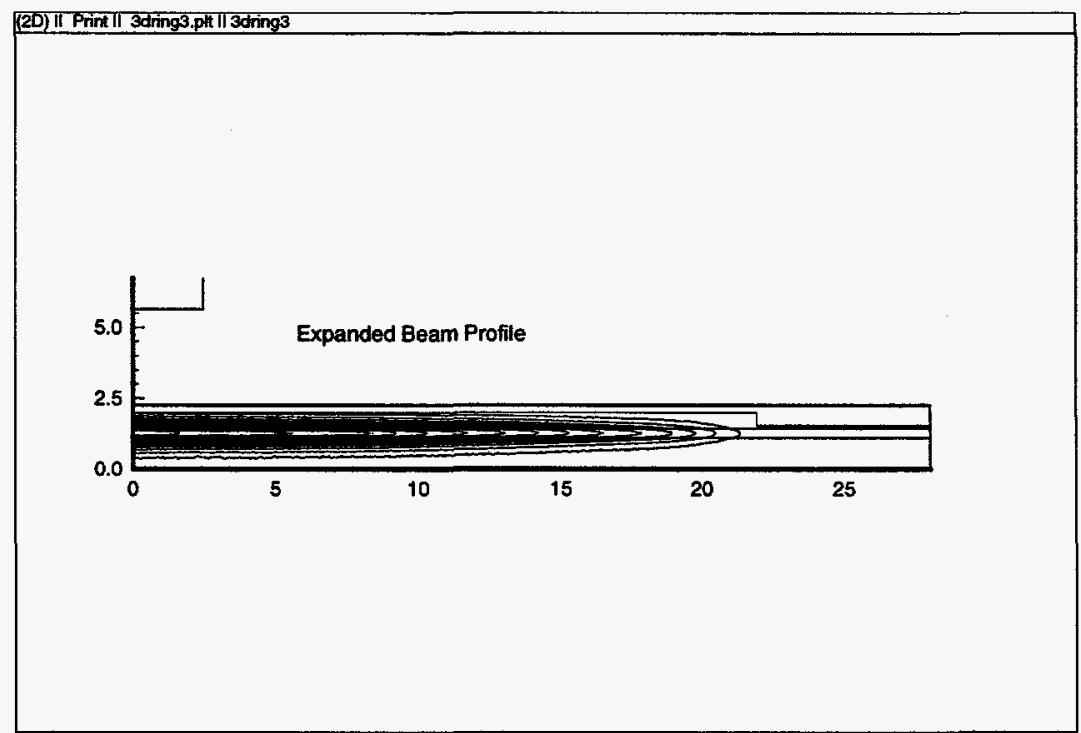

Fig. 4 Near-field intensity profile (to scale) of the input beam (Fig. 2) after propagating $1000 \mathrm{~mm}$ down the flared waveguide. Dimensions are in microns. The accompanying far-field pattern is shown in Fig. 4.

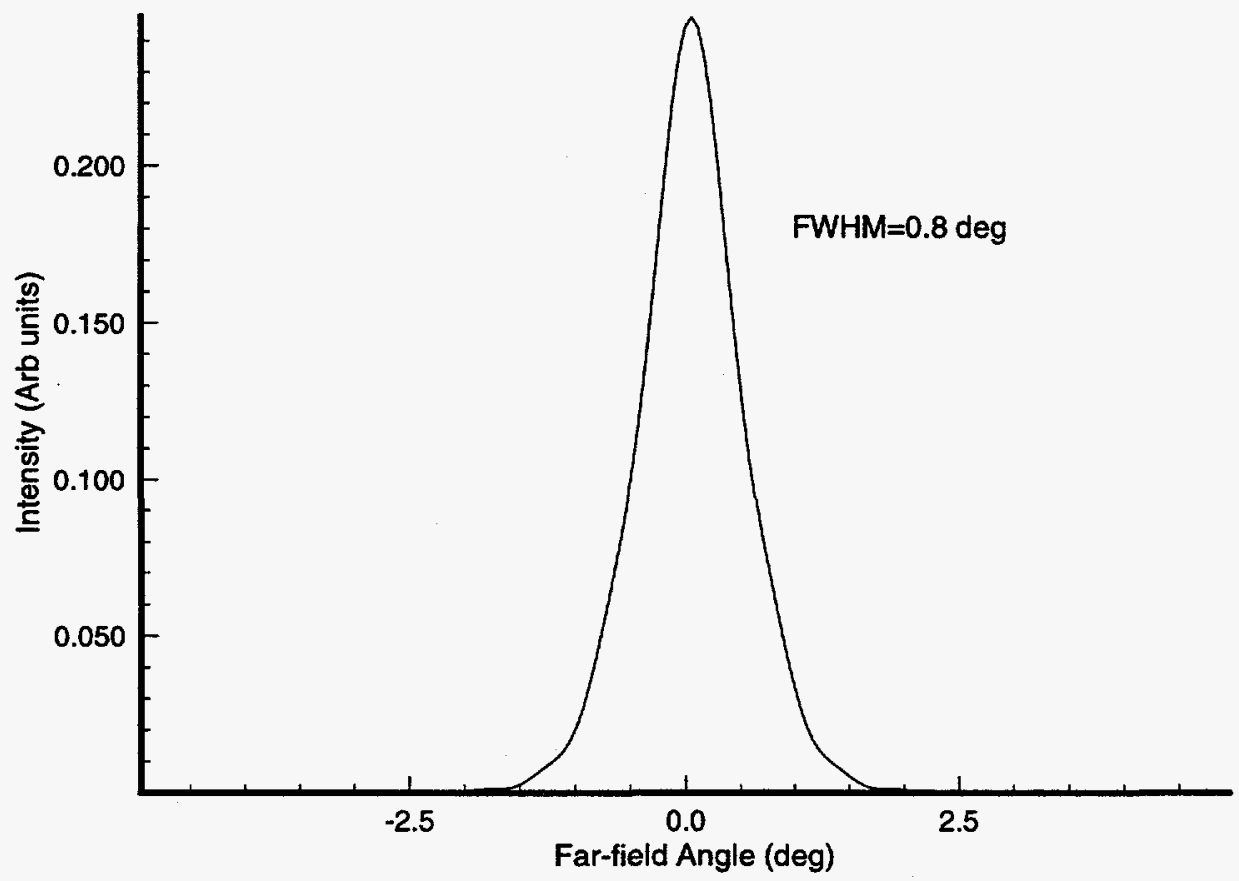

Fig. 5 Far-field pattern of the expanded beam whose near-field profile is shown in Fig 4. The beam divergence in air should be about 3.3 times the value shown in the Fig.. 


\section{Grating Design}

Design of the surface-emitting grating was pursued with the following criteria in mind:

1. Emission should be as close to normal as possible.

2. High efficiency is crucial (The efficiency we are referring to here is total power radiated into the desired direction / total incident power.)

3. The grating should be made as short as possible consistent with 2 in order to keep the overall beam aspect ratio as close to unity as possible.

The basic approach is to etch a second-order grating into the top of the flared waveguide as shown in Fig. 3. However, a simple grating will reflect the majority of the light into the substrate. Since the decision was made by the group to take light out the top of the wafer rather than through the substrate, a blazed grating is required, oriented such as to favor emission into the air. Even this modification still resulted in substantial amounts of light propagating into the substrate, thus necessitating the addition of a quarter-wave stack epitaxial reflector. A short section of the resulting composite structure was modeled using finite-difference solution of the 2-D Helmholtz Equation, with the geometry shown in Fig. 6 . Notice that Fig. 6 is not drawn to scale, and the reflector stack is not drawn in detail, but rather indicated schematically. The actual stack used in the calculations consisted of alternate layers of GaAs and AIAs, each $0.107 \mu \mathrm{m}$ thick. Exact quarter-wave thicknesses were not used due to mesh size limitations. Also, only 11 layers were used in order to limit computer runtimes. Such a stack is only $50 \%$ reflective, but serves to demonstrate proof of principle. Details of the desired stack geometry are given in the Appendix.

The pitch of the grating has been chosen to be $0.3825 \mu \mathrm{m}$, slightly less than the second-order value of $0.3966 \mu \mathrm{m}$ (based on a modal index of 3.328). This detuning serves to keep the guided mode reflection coefficient low $(<2 \times 10-3)$. However, it also results in emission slightly off of normal, as shown by the calculated far-field pattern in Fig. 7. Note that the angular width shown in Fig. 7 is indicative of a $17 \mu \mathrm{m}$-long grating, and will be considerably more narrow when emitted from a full-length grating.

The power emission profile for a full-length grating is determined from the numerical results by using the latter to determine the coefficient in a simple exponential law expression. The power left in the guided mode for the grating shown in Fig. 6 is given by

$$
\frac{P}{P_{0}}=e^{-303.4 z}
$$

According to the above equation, over $94 \%$ of the light is removed from the guided mode after a distance of $100 \mu \mathrm{m}$. However, it is recommended that the 
grating be made $200 \mu \mathrm{m}$ long as a precaution. Of the resulting radiated power, approximately $90 \%$ is predicted to be in the preferred direction when a full-width reflector is used, giving overall efficiencies as high as $85 \%$.

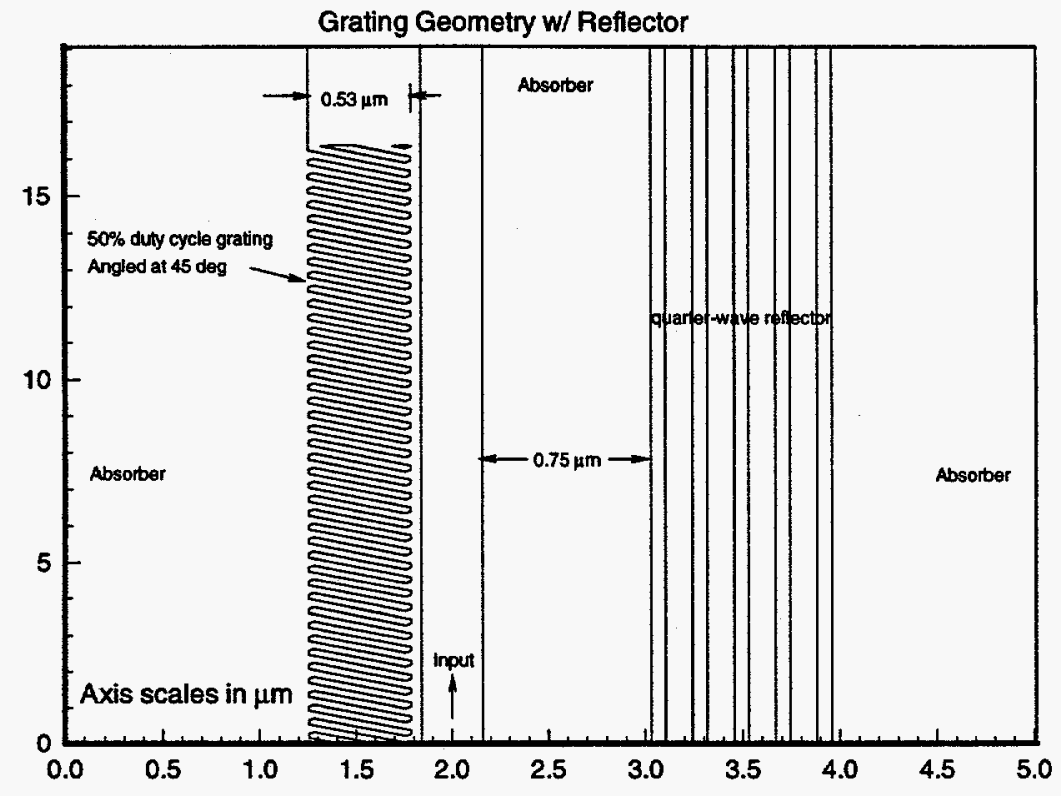

Fig. 6 Geometry used for model calculations of grating emission (not to scale). The reflector is only shown schematically, as explained in the text.

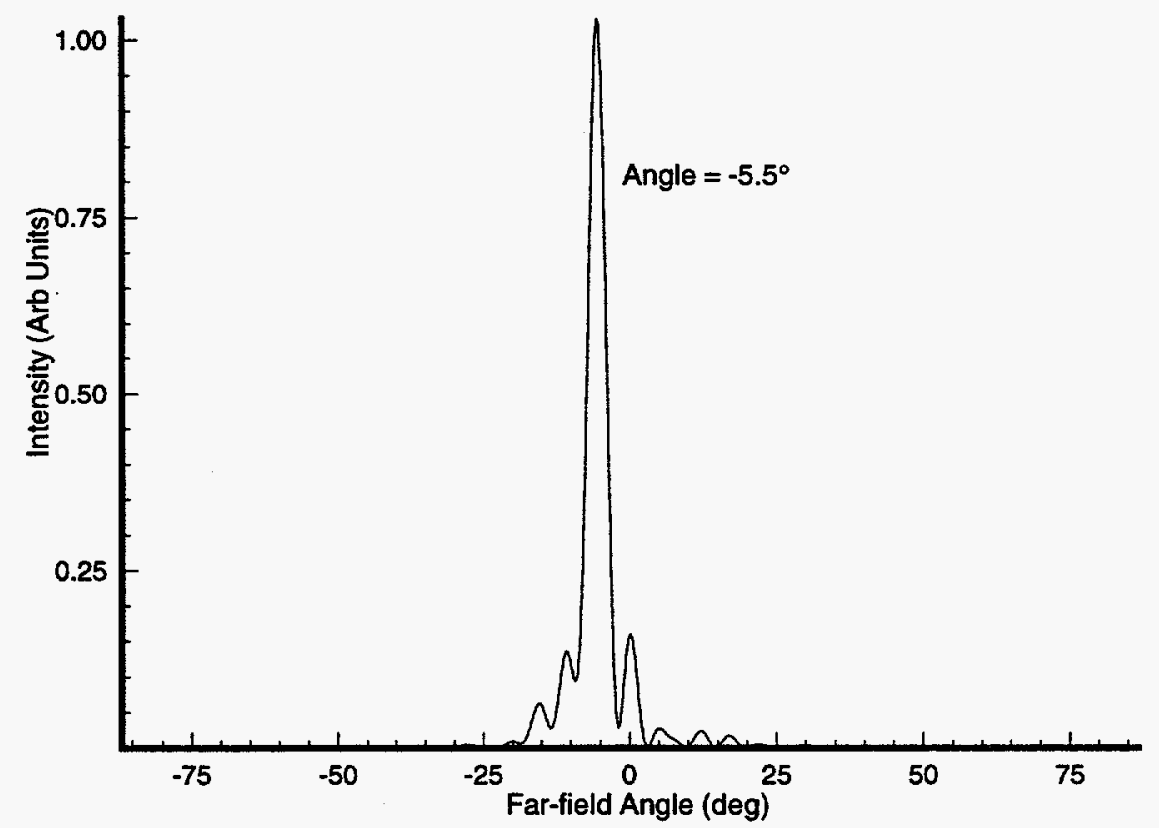

Fig. 7 Predicted far-field emission from grating into air. Detuned grating emits a few degrees from normal. 


\section{Fabrication of Grating Couplers}

Theoretical results were translated into a design and masks for both grating and mirror output couplings. Growth structures in GaAs have been received and mirror reflectance and layer thickness have been evaluated. The gratings were fabricated using direct electron-beam lithography on the JEOL JBX-5DII, with subsequent levels defined with conventional contact printing. The lithography required to delineate the expansion and, in particular, the boundary between expansion and grating, was laid out with special care to provide very smooth sidewalls for the expansion. The expansion was digitized using two data types: one to define a dose adequate to clear resist from all but the edges, and a second to define just the outer $0.5 \mu \mathrm{m}$ edge of the feature. The edge dose was adjusted $10 \%$ higher than the general area dose to provide very smooth vertical resist walls. To accommodate the proximity effect resulting from the widest portion of the expansion mated with the grating of submicron periodicity, the grating was digitized as single-pass zero-width lines, using three data types to taper dose as the grating approached the mouth of the expansion. Proximity effect resulted in a dose $50 \%$ lower for the lines adjacent to the expansion than the dose required for lines $6 \mu \mathrm{m}$ away from the expansion.

The pitch of the gratings is $0.3825 \mu \mathrm{m}$, with a $50 \%$ duty cycle. The grating was patterned on PMMA, which served as a lift-off mask for titanium lift-off. The titanium was used as a reactive-ion beam etch (RIBE) mask for a subsequent oxygen etch through the thick tri-level resist. The tri-level resist served as a mask for a chlorine RIBE etching.[2] The dramatic effects of etch depth will be presented. Gratings have been made at both $90^{\circ}$ and $45^{\circ}$ angles to the etch beam. The latter etches were used to blaze the gratings.

The entire test structure consisted of three features, a straight waveguide, an adiabatically expanded waveguide, designed to be cleaved for evaluation, and an adiabatically expanded waveguide with gratings etched into it.(Fig. 8).

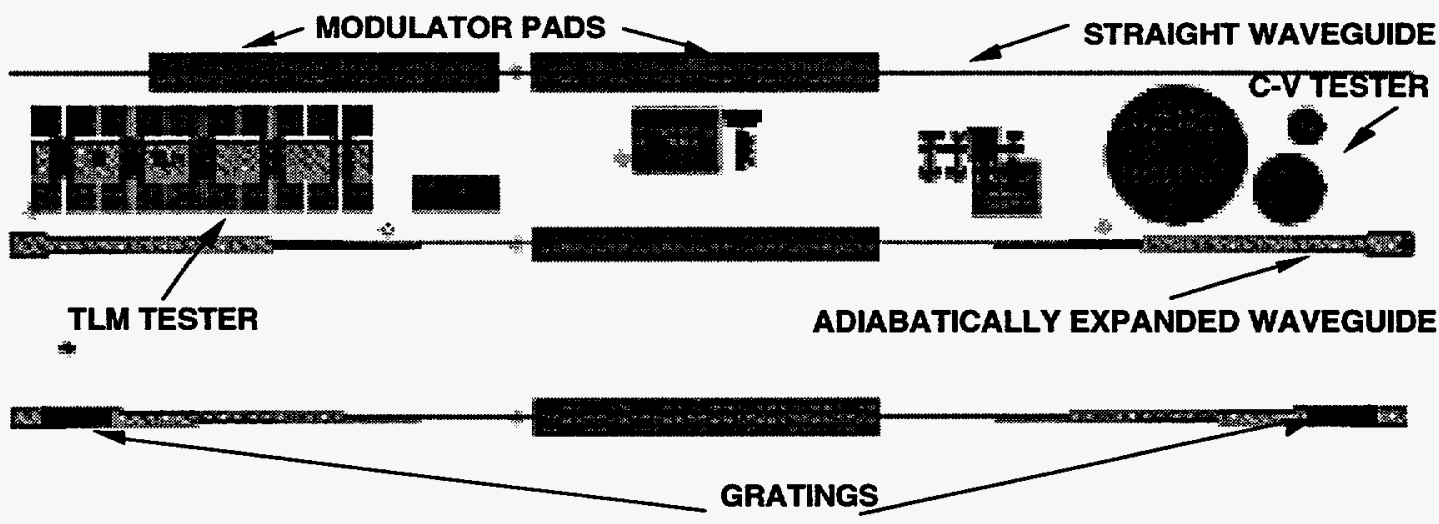

Fig. 8. Test structure for development of advanced packaging technology 
All devices were planarized with polyimide and portions of the waveguide were metallized to provided a modulator structure. The modulators could be biased to observe any possible effects of voltage on the structure and to make it easy to discern modulated output light and to aid in loss measurements. An SEM of the vertical grating with Bragg reflectors is shown is Fig. 9.

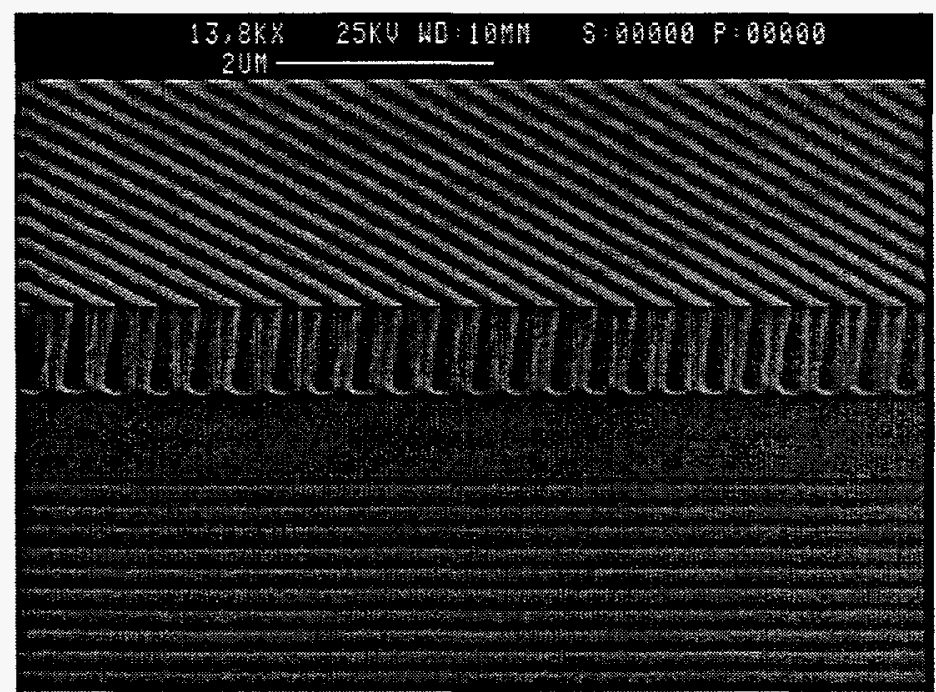

Fig. 9. Vertical Gratings with Bragg Reflectors

The above design predictions were corroborated by far-field measurements obtained by examination of the rectangular-shaped image at various distances from the wafer (Fig. 10). These measurements indicate a beam divergence of $3^{\circ}$ normal and $1^{\circ}$ parallel to the waveguide. An improvement to the grating designed has been fabricated. This improvement consists of a $45^{\circ}$ blaze of the gratings (Fig.11).

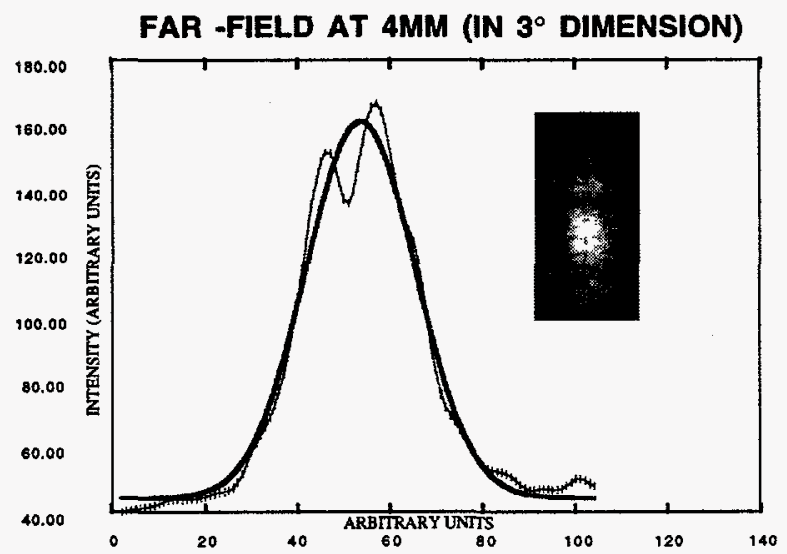

Fig. 10. Far-field measurements of the beam exiting the grating. 


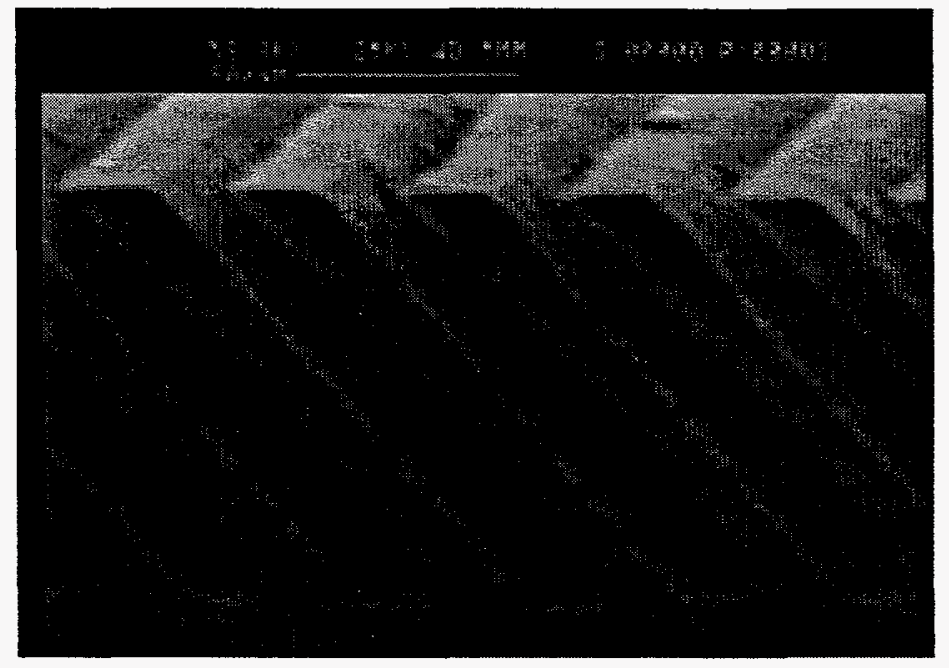

Fig. 11. 45 degrees blazed gratings.

The purpose of this blaze is to change the ratio of light directed up from the grating compared to that going down into the substrate. This should improve grating output efficiency. Improvements in binary optic design have been made, using previous measured results. The most important improvement is the development of an off-axis design.

\section{Binary Optic Lens Design and Fabrication}

All of the diffractive lenses for this project have been designed with software developed at SNL. The design algorithm is based on Fermat's principle, solving for the roots of the nonlinear equations describing the phase relationship between the rays traveling from different points on the lens surface to the focal points. Lenses fabricated for this project were diffractive elements that have stair-step approximations to the Fresnel lens profiles. These lenses are formed by 3 repeated mask and etch steps to form 8 phase levels in a process often referred to as binary optics. The first lenses were fabricated in silicon by a combination of optical lithography and reactive ion beam etching. The first lenses were on-axis designs that needed to be positioned with the surface of the lens perpendicular to the direction that the beam exited the waveguide grating coupler. An SEM of the Binary optic lens is shown in Fig. 12. 


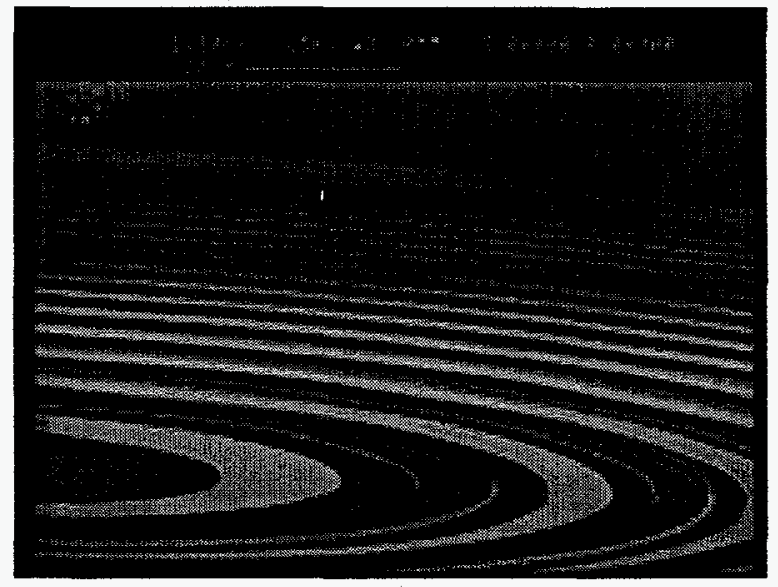

Fig. 12. A scanning electron micrograph of a silicon 4 phase-level binary optic lens.

Because the beam from the grating coupler was not circular, the lens designs included anamorphic designs in which the lens had different focal lengths for orthogonal transverse axes to transform the beam to a circular focal spot for more efficient coupling into the fiber. These lenses performed well as related in the experimental results. For our second series of lens designs, we wanted to transform the beam direction as well as its shape so that the lens could be located on a plane surface parallel to the waveguide chip surface, but not perpendicular to the output beam of the waveguide grating coupler which not normal to the grating surface. This required design of a sophisticated diffractive lens combining off-axis performance with the anamorphic focal properties.

These lenses were fabricated using electron-beam lithography to improve the level to level alignment in fabrication and to better reproduce the fine features required for good off-axis performance (Fig.13). Unfortunately, the gratings for which the lens was designed were destroyed in testing and it was not a good match for the properties of the remaining grating couplers. In addition, overall diffraction efficiency of the off-axis lens was not as good as the on-axis lenses. When new high-efficiency waveguide grating couplers are available, we plan to redesign this lens for the beam properties of the new couplers and changes have been made in the lens design algorithm that should yield better diffraction efficiency.

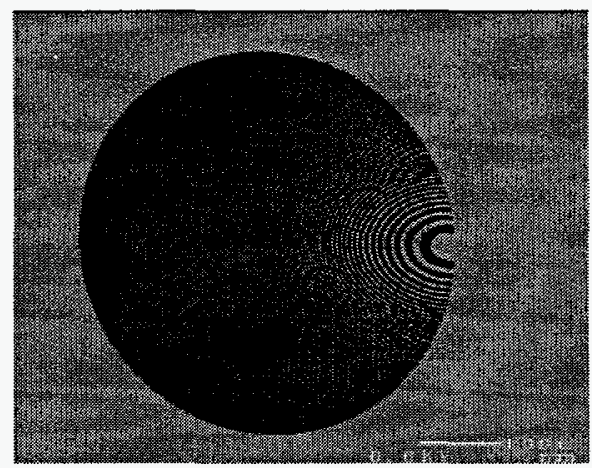

Fig. 13. Anamorphic binary optic lens. 


\section{Microwave Launch to Photonic Device}

The design of microwave structures to launch to photonic devices vary from either a microstrip or a coplanar launch. The use of coplanar and coaxial microprobes for semiconductor devices has made microwave measurements a lot easier to make with better accuracy. Microstrip and coplanar photonic devices were considered for designing the package transitions from coaxial to microstrip to coplanar transmission lines. Using commercially available coaxial connectors along with custom package designs, high frequency fixtures up to $50 \mathrm{GHz}$ where built. The microstrip transmission line connecting the coaxial connectors to the photonic device was made of RT/duroid (Trademark). This material has a dielectric constant of 2.2 and a loss tangent of .0009 . The microwave low loss properties of this material allowed us to get low insertion loss at frequencies from dc to $50 \mathrm{Ghz}$.

An $18 \mathrm{~mm}$ long microstrip transmission line on duroid launched to $V$-connectors at both ends was assembled in a an aluminum housing. Results of sparameters made on the automatic network analyzer are shown in Fig. 14. The graph shows the return loss is better than $10 \mathrm{~dB}$ from $45 \mathrm{MHz}$ to $50 \mathrm{GHz}$ and an insertion loss of $1 \mathrm{~dB}$ at $50 \mathrm{GHZ}$. Therefore we highly recommend that duroid be used for high frequency applications where low insertion loss is needed. Also, duroid is easy to pattern and to cut to the desired length.

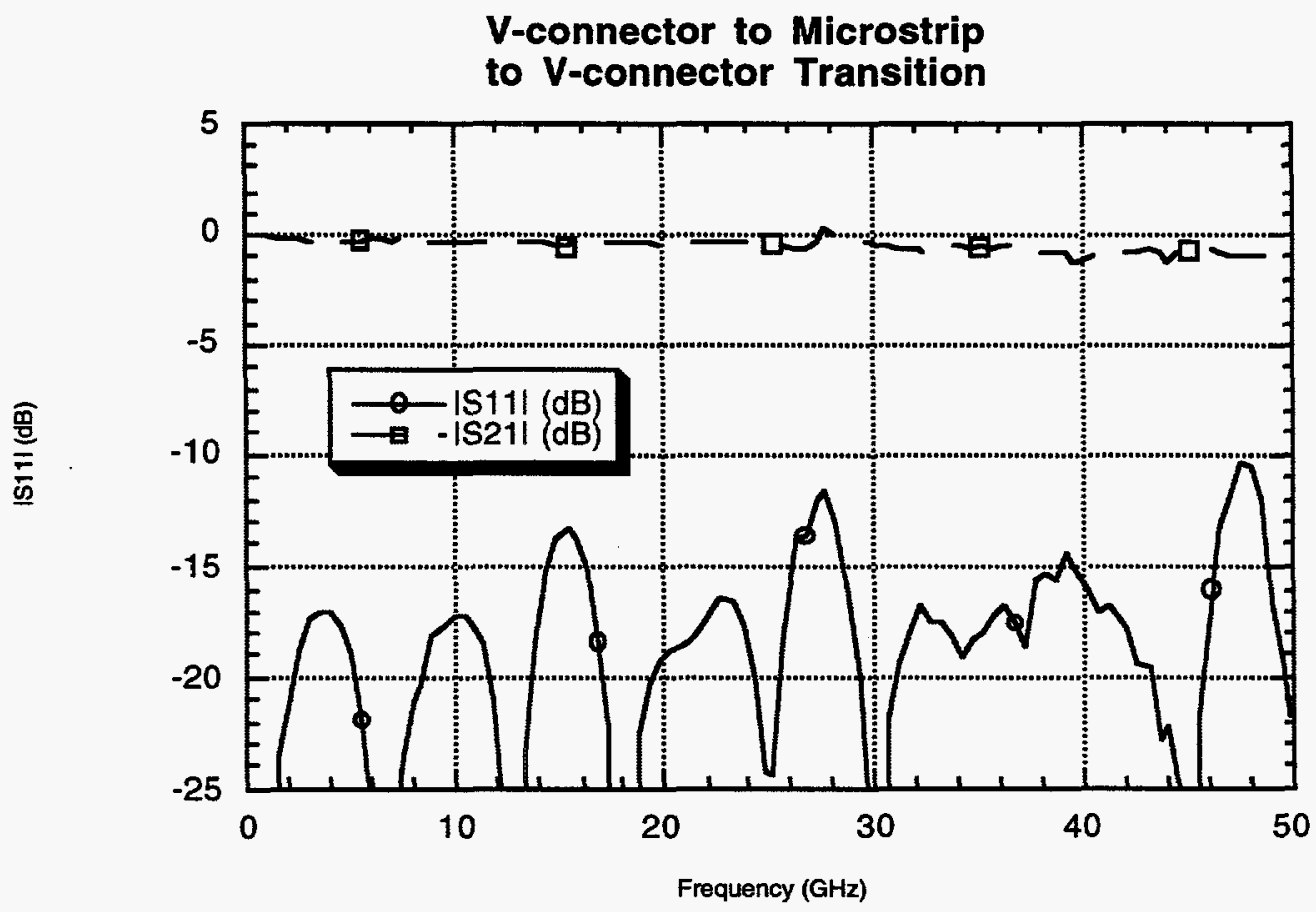

Fig. 14. Insertion loss and retum loss of microstrip to V-connector launch 
A coplanar to microstrip transition was used for coplanar photonic designs. These transitions were commercially available from Probepoint (Trademark) and they allowed low loss transitions up to $50 \mathrm{Ghz}$. In order to find out what the losses would be when launching from V-connector-to-microstrip-to-coplanar a mechanical PIC was assembled and mearsured as a thru on the automatic network analyzer. Results of S21 shown in Fig. 15 indicate an insertion loss of about $1.7 \mathrm{~dB}$ when subtracting the loss of the PIC at $40 \mathrm{Ghz}$. Once again, this shows that these microstrip-to-coplanar transitions along with microstrip duroid are suitable for high frequency applications.

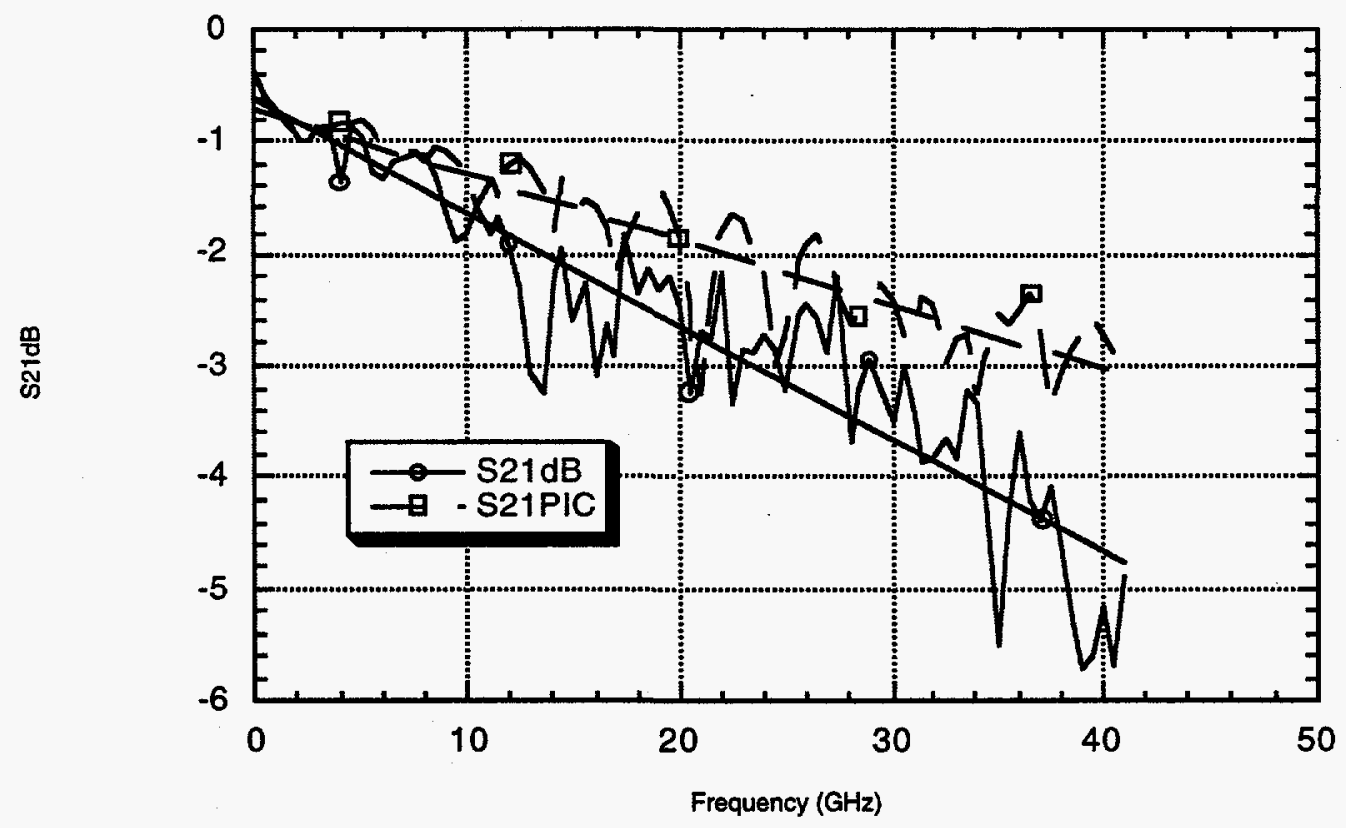

Fig. 15. S21dB of PIC loss and S21dB of PIC launched to coplanar transition and V-connectors.

\section{Packaging Assembly}

\section{A. V-Grooves in silicon}

Silicon substrates 'holders' with v-grooves were produced for precision placement of optical fibers. It was envisioned that the holders could be used both for mating to optical sources or for use in holding fibers for end polishing. Designs of 250 micron and 500 micron spacing for groups of fours was incorporated in the mask set. To compensate for slight variations in fiber 
diameter, minor changes in the groove width was also included. A total of eight variations were incorporated in the mask design. Fig. 15 shows the calculations made to determine the correct size of the v-grooves needed for this fiber. In Fig. 16 a SEM photo of a fiber in a $v$-groove is shown.

The lot definition for producing the holders was done in conjunction with the production of substrates for stacked multi-chip modules (MCM) which required additional "back side" patterning. Both the single sided and double-sided patterning of wafers were performed on a Suss contact alignment system using IR to image through the wafer. Unique alignment targets patterned in metal on the front side are utilized for good target recognition when aligning the backside. A special IR video imaging system is employed which allows the operator to view the front side, which is face down on the chuck, while moving the back side into alignment under the mask. The single sided v-groove mask levels supported APPA. The back-side mask level supported stacked MCMs. This double sided patterning technique was a new capability for the Microelectronics Development Laboratory (MDL).

The v-grooves etch process was accomplished by a selectively etch into the silicon substrates using a thin film mask made from thermal silicon nitride. A $\mathrm{KOH}$ solution was used to preferentially etch the silicon faster along the $\langle 111\rangle$ plane, giving a 54.7 degree angle groove. Depth of etch is determined by the etch rate of the solution and time. The groove etch is 'self stopping' when allowed to reach the full depth. However, since the fiber rests on the edges of the groove, it is only necessary to carry the etch to a depth which clears the fiber radius. A partial etch facilitates the use of thinner silicon, especially when two sided etching is being performed. Mechanical strength is also better with the partial etch.

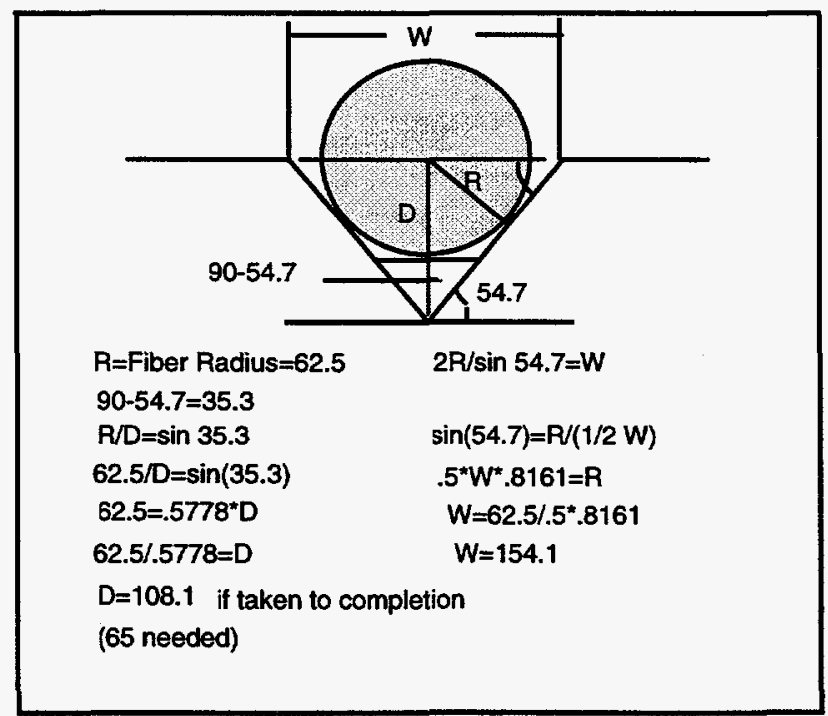

Fig. 16. Calculation of width $\mathrm{W}$, and distance $\mathrm{D}$ for $62.5 \mu \mathrm{m}$ fiber.

Dimensions are in microns. 


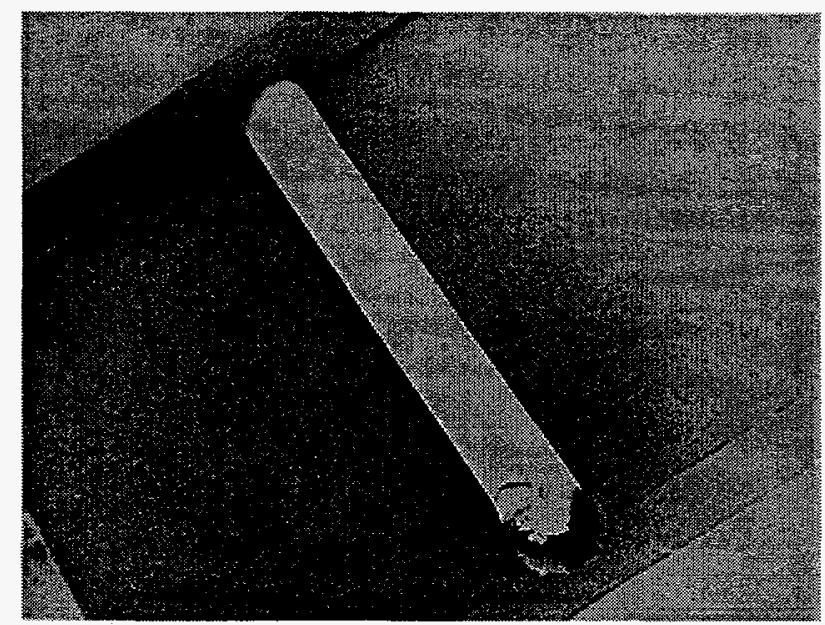

Fig. 17. SEM of single-mode fiber in a silicon V-groove

\section{B. Laser Drilling of Vertical Vias in Silicon}

In order to position the optical fiber directly over the beam input and output location, a silicon holder was made with holes drilled using a laser operating at $532 \mathrm{~nm}$.[3] An optical Tre-pan assembly rotates the beam to achieve circular holes. The focal length lens was $40 \mathrm{~mm}, \mathrm{Q}$-switch repetition rate $=4 \mathrm{KHz}$, with $5.8 \times 10 E 9$ watts $/ \mathrm{sq} \mathrm{cm} .150$ nsec pulse length. The focused spot size was approximately 12 microns. The wavelength is achieved by the use of second harmonic generation from a Nd:Yag laser $(1064 \mathrm{~nm})$. The holes made at this wavelength and settings are created by a mechanism of vaporization and ablation to remove the silicon material (Shown in Fig. 17). The interior walls of the holes exhibit no silicon melt residue. Oxide powder is the only product left on the surface. This is easily removed in a D.I.water ultrasonic rinse.

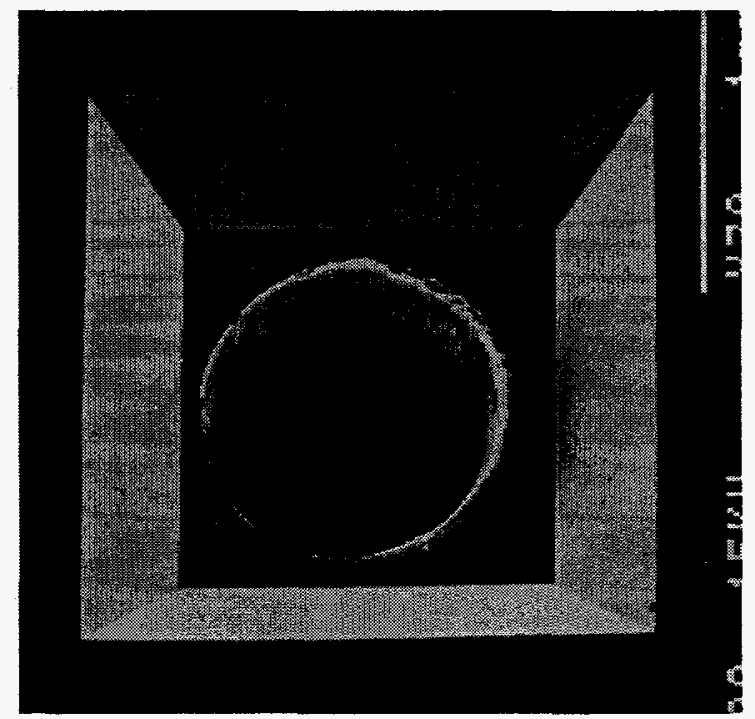

Fig. 18. SEM of laser drilled hole on a silicon substrate on an etched window. 


\section{Assembly}

A silicon submount, patterned with alignment location markings was used as a carrier for the grating chip. Silicon was chosen as a transition material to reduce stress which might occur due to the difference between the expansion rates of the GaAs chip and the aluminum housing. The grating chip was bonded to the silicon submount using Staystick 301 insulating paste adhesive, dispensed by hand, and cured at $150^{\circ} \mathrm{C}$ for 10 minutes. Using an IR microscope, the chip and mount were located in the housing prior to their being bonded to the aluminum using the same adhesive and cured at $250^{\circ} \mathrm{C}$ for additional $10 \mathrm{~min}$. Following a determination of the angle and distance between the input and output of the beam through the grating, holes were laser drilled into silicon at the corresponding distance. Two pieces of silicon with exact positioned laser drilled holes were stacked to act as a holder for the fibers. The double silicon (.052" thick) and fibers were bonded using Staystick 373 cured at $250^{\circ} \mathrm{C}$ and $150^{\circ} \mathrm{C}$ respectively. A mechanical packaged assembly is shown in Fig. 18.

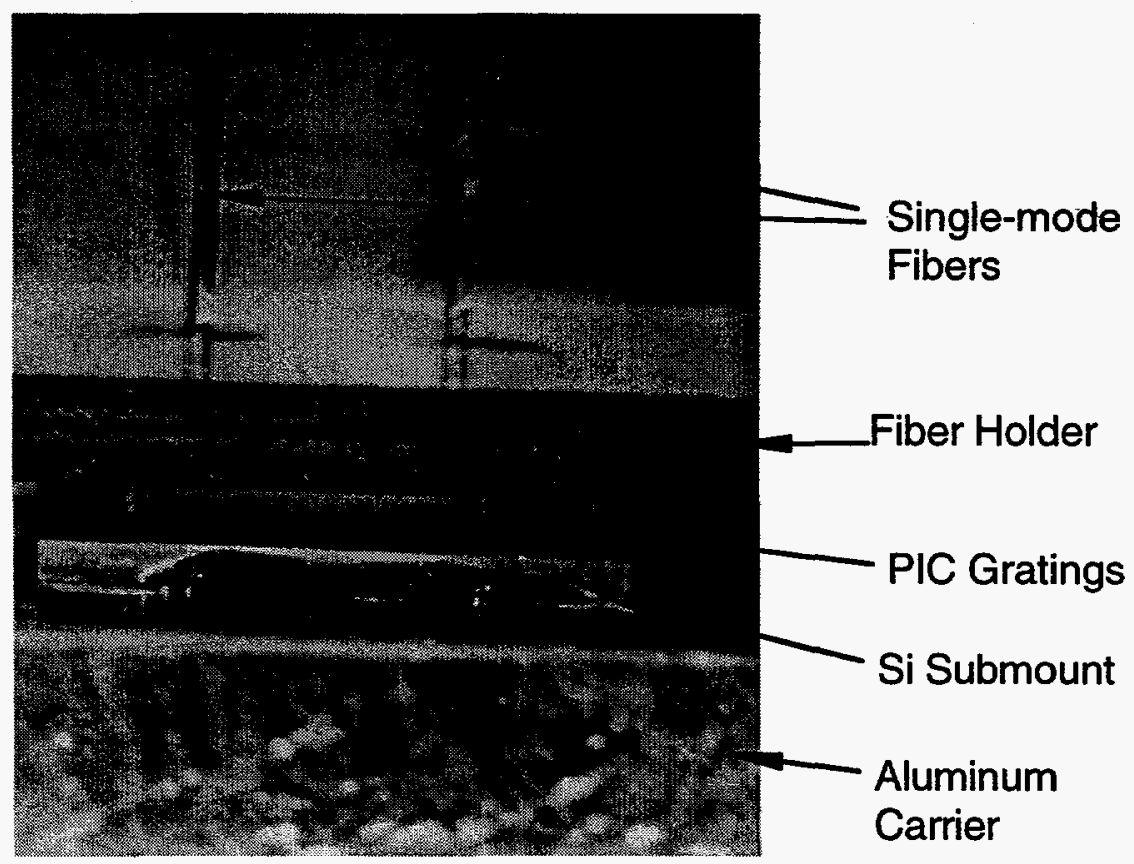

Fig. 19. Complete mechanical assembly of packaged APPA concept.

\section{Results}

The package concept was demonstrated as illustrated in Fig. 4. A 1.3 micron diode-pumped YAG laser was coupled via a polarization-preserving singlemode fiber into a GaAs/AIGaAs waveguide on a fabricated test chip. The light was then coupled out of the chip surface by the adiabatic taper and grating and collected by a silicon microlens $2 \mathrm{~mm}$ away. The silicon microlens focused the 
light into the same type of single-mode fiber. The waveguide losses, grating output efficiency, and microlens diffraction efficiency were all measured independently. The total loss through the system was $-18.5 \mathrm{~dB}$. Neglecting the $-8.8 \mathrm{~dB}$ loss for butt-coupling into the waveguide, as measured by OTDR, the coupling loss through the waveguide-grating-lens-fiber route was $-9.7 \mathrm{~dB}$, including a guiding loss of $-2.3 \mathrm{~dB}$ in the semiconductor waveguide. The efficiency of coupling through the silicon lens into the fiber was $30 \%(-5.2 \mathrm{~dB})$ after emission from the grating.

We believe this is a promising indication of the potential for this application of diffractive optics to packaging of complex PICs. Some obvious areas of improvement are improving the diffraction efficiency of the silicon microlenses and optimizing the lens design for coupling of the grating output into the fiber mode. The coupling data indicates the anamorphic lenses bracketed the optimum configuration for this application and improvement is expected for the next generation. The off-axis emission of the grating is necessary because of the need to detune the grating to eliminate feedback problems for the PICs. Consequently, the next lens designs will include optimization for off-axis performance so they can be positioned with their surface parallel to the chip surface as required for the packaging concept.

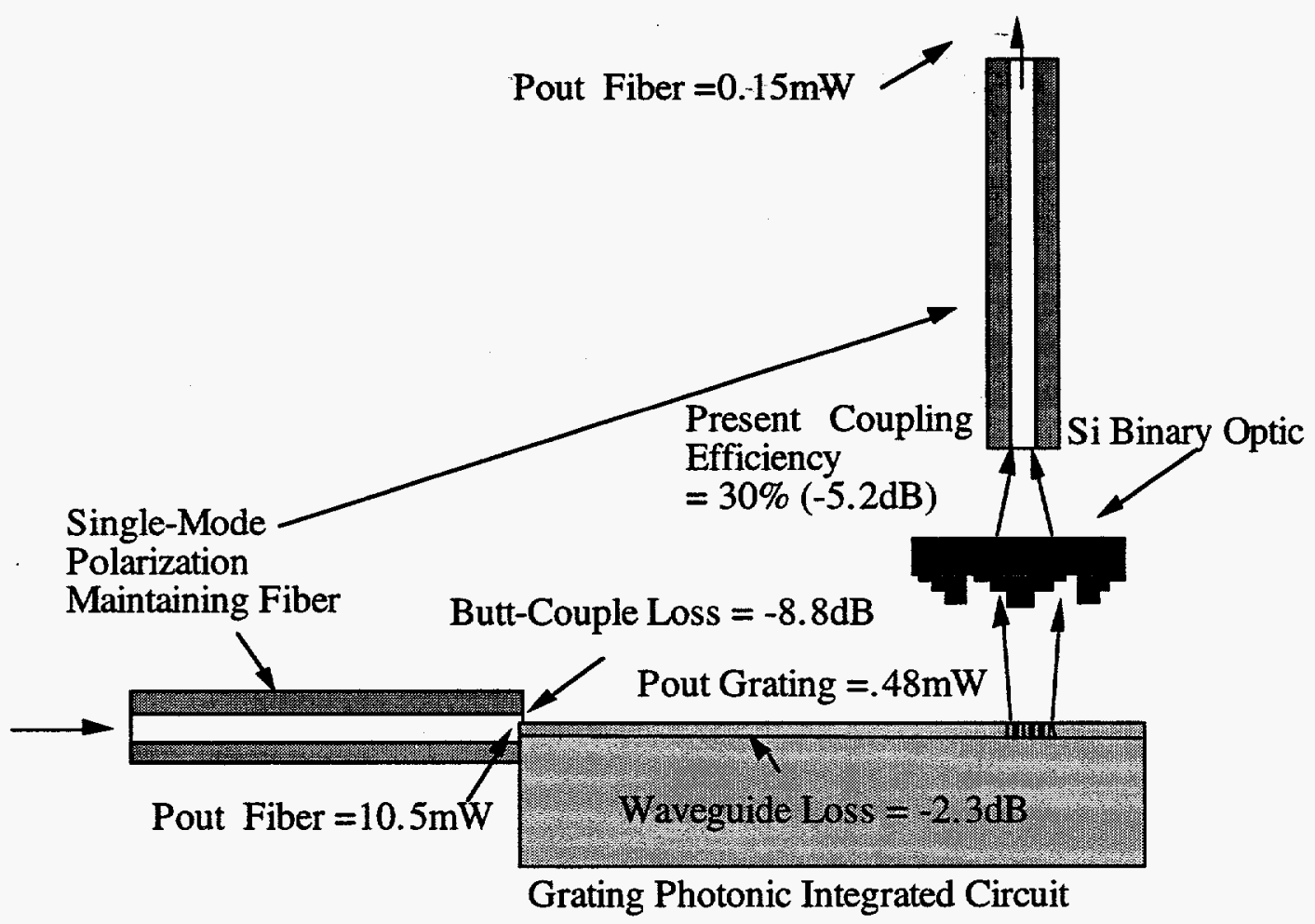

Fig. 20. Configuration for coupling from ridge waveguide to singlemode fiber via waveguide grating-coupler and silicon microlens. 


\section{Conclusion}

An advanced photonic integrated circuit package has been demonstrated. A coupling efficiency of $30 \%$ has been achieved. Extensive design, fabrication and testing of the optical and electrical components has been demonstrated. These concepts are currently being used to design new products. One useful application that may also benefit future testing of PICs is the ability to do high frequency on-wafer probe optical waveguide measurements for phase and amplitude modulators(Fig. 20). This will help to determine known good die before individual die optical testing or packaging assembly is done.

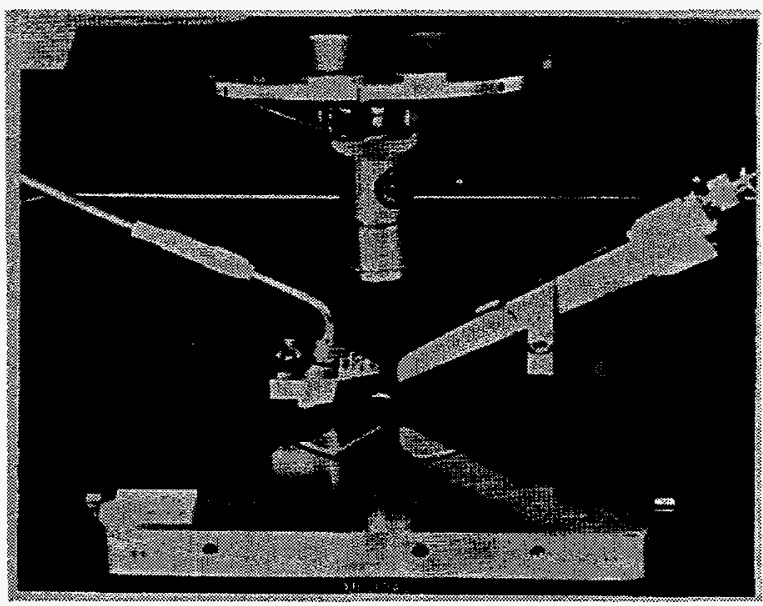

Fig. 21. On-wafer optical fiber probe 


\section{References}

[1] S. H. Kravitz, V. M. Hietala, G. A. Vawter (1322) and W. J. Meyer (1342), Phased-Array Antenna Control by a Monolithic Photonic Integrated Circuit, COMPASS, SPIE, Boston, MA, 9/3-6/91.

[2] G. A. Vawter, J. F. Klem, G. R. Hadley, S. H Kravitz, "Highly accurate etching of ridge-waveguide directional couplers using in situ reflectance monitoring and periodic multilayers", Appl. Phys. Lett. vol. 62 (1), 4 January 1993.

[3] SAND93-1772. UC-704 Laser Drilling of Vertical Vias in Silicon, W. D. Miller, R. A. Gassman and D.M. Keicher. 


\begin{abstract}
Appendix A. Reprint of 1994 Integrated Photonics Research Leeting, San Francisco, CA, 2/17-19/94 Tech. Digest Series 3 . P. 42 .
\end{abstract}

Numerical simulation of Reflecting Structures via solution of the 2D Helmboltz Equation*

\author{
G. Ronald Hadley \\ Sandia National Laboratories \\ Albuquerque, New Mexico 87185-5800 \\ (505) 844-4015
}

ABSTRACT

A method is described for modeling two-dimensional reflecting structures based on a solution of the scalar Helmholtz Equation. The latter is solved using an alternating-direction-implicit iterative method together with a semi-optimum sequence of acceleration parameters similar to those introduced decades ago for the solution of elliptic equations with positive-definite operators. The resulting technique is efficient, simple to program, allows the simulation of complex structures with modest storage requirements, and is of very general applicability.

*This work performed at Sandia National Laboratories, supported by the U. S. Department of Energy under contract number DE-AC04-76DP00789. 
The rapidly-growing science of photonics continues to introduce new structures to serve as building blocks for optical circuitry. As before, the high development cost of these structures provides ample justification of the need for numerical models that can help eliminate guesswork and optimize designs. For structures characterized by more or less unidirectional propagation, beam propagation methods have proven to be very useful for this purpose, and of general applicability. However, numerical methods for structures that generate reflections tend to be complicated to implement, and of limited scope, with every new problem requiring a new method. For example, bidirectional beam propagation ${ }^{1}$ and eigenvalue expansion techniques ${ }^{2}$ work well for facet reflectivity calculations, but are unable to treat second-order gratings. By the same token, theories developed for such gratings ${ }^{3,4}$ are specific to that type of problem and therefore useless for any other structure (in addition to being highly complex and difficult to implement).

In contrast, the scalar Helmholtz Equation accurately describes the response of a general two-dimensional structure to incident light of a single frequency and polarization, including the effects of reflections from dielectric interfaces as well as propagation at any angle. It thus can be used to investigate the behavior of a very large class of passive structures. Its previous usefulness for this purpose has been severely restricted due simply to the difficulty of solving the associated difference equation. This difficulty arises both from the necessity of fine zoning (since no slowly-varying envelope approximation can be employed) and the fact that the difference operator is not positive-definite, thus rendering many of the time-honored methods for 
solving elliptic difference equations either totally unusable or prohibitively slow.

This letter describes a technique for overcoming this difficulty based on an alternating direction implicit (ADI) solution procedure employing near-optimum acceleration parameters similar to those introduced decades ago by Wachspress ${ }^{5}$ for other elliptic equations with positive definite operators. This procedure is much simpler than other iterative techniques ${ }^{6}$, is relatively fast even for very large problems, and requires minimal storage. Most importantly, however, it is extremely general and can therefore be successfully applied to a great variety of structures with a minimum of programming effort.

This method is intended to model "two-dimensional" structures that do not mix polarizations. An analysis of the single frequency response of such a structure is provided by the scalar Helmholtz Equation

$$
\nabla^{2} A+\varepsilon(x, z) A=0
$$

where $\varepsilon(x, z)$ is the complex dielectric constant, and the coordinates $x, z$ have been normalized via multiplication by the vacuum wavevector $k_{0} \equiv 2 \pi / \lambda_{0}$. Equation (1) is to be solved in the rectangular region shown in Fig. 1. Here an incident mode enters through the bottom boundary (usually an eigenmode of an input waveguide), encounters some structure in the interior where it scatters in various directions, and eventually reaches the absorption regions positioned near the boundaries. For those calculations presented here, the bottom Dirichlet boundary condition describes the incident mode. It can be easily shown that, for light scattered backwards from the problem interior, this boundary is therefore perfectly reflecting for all other modes, but perfectly 
transmitting for the incident mode. This allows an easy calculation of the reflection coefficient for that mode by examining the phase of the wave at the first row of mesh points in from the boundary. The field amplitude $A$ in Eq. (1) represents the $y$-component of $E(H)$ for TE(TM) polarizations.

Equation (1) is next discretized on a mesh of sufficient resolution so that $\Re(\sqrt{\varepsilon}) \Delta\left[\begin{array}{l}x \\ z\end{array}\right] \leq 0.4$ in order to maintain good accuracy. Solutions of (1) on this mesh are obtained using the well-known iterative ADI procedure7

$$
\begin{aligned}
& \left(\omega_{n}+\delta_{z}^{2}\right) A^{n+\frac{1}{2}}=\left(\omega_{n}-\delta_{x}^{2}\right) A^{n} \\
& \left(\omega_{n}+\delta_{x}^{2}\right) A^{n+1}=\left(\omega_{n}-\delta_{z}^{2}\right) A^{n+\frac{1}{2}}
\end{aligned}
$$

where $\omega_{n}$ is a complex acceleration parameter that is allowed to vary from step to step and is chosen to optimize convergence. The difference operators are defined by

$$
\begin{aligned}
& \left.\delta_{x}^{2} A\right|_{i, j} \equiv \frac{A_{i+1, j}+A_{i-1, j}-2 A_{i, j}}{(\Delta x)^{2}}+\frac{\varepsilon_{i, j}}{2} A_{i, j} \\
& \left.\delta_{z}^{2} A\right|_{i, j} \equiv \frac{A_{i+1, j}+A_{i-1, j}-2 A_{i, j}}{(\Delta z)^{2}}+\frac{\varepsilon_{i, j}}{2} A_{i, j}
\end{aligned}
$$

When the iterative method just outlined converges, it is guaranteed by the form of Equations (2) to converge to a solution of the Helmholtz Equation (1). The method will in fact converge if the problem region contains absorption and the parameters $\omega_{n}$ are properly chosen. This problem was first addressed for the case of positive definite 
operators by Wachspress ${ }^{5}$, and simple analytic formulas presented for optimum convergence using arbitrarily long sequences of acceleration parameters. Although some progress has been made in extending this treatment to cover operators with complex eigenvalues ${ }^{8}$, the present case has not yet been solved exactly. Analytic solutions for optimum convergence employing a single parameter $\omega$ may be determined for the case of uniform absorption. However, one parameter solutions are not particularly useful due to their typically slow convergence rates. Consequently, an alternating sequence of two parameters $\omega_{1}, \omega_{2}$ is necessary, representing an approximate optimization, in order to provide rapid convergence. Although their derivation will not be presented here due to space limitations, it may be shown that good convergence rates for a wide variety of problems may be obtained by setting $\omega_{1}=(-200,0$. and $\omega_{1}=(0.05 b, 1.1 b)$ with $b=\Re(\varepsilon / 2)$ for some representative value of $\varepsilon$. These parameters are expected to hold for problems utilizing the zoning criteria mentioned previously, and where absorbers are employed primarily around the problem periphery.

The ADI method just described has some distinct numerical advantages that make it an attractive alternative even for large problems: (1) The use of semi-optimum acceleration parameters as presented above provides good convergence in 200-600 iterations for most problems, resulting in reasonable overall runtimes. (2) Storage requirements are proportional to the number of mesh points $\boldsymbol{N}$ employed (in contrast to direct solution methods). (3) The values of the optimum acceleration parameters for the two-parameter sequence are relatively insensitive to the problem type and mesh size. (4) The ADI method is very simple to code in comparison with other methods. 
Because of its similarity to the scalar finite-difference-timedomain (FDTD) method ${ }^{9}$, some discussion comparing the two is warranted. The latter method solves the time dependent wave equation, which reduces to Eq. (1) in the harmonic limit. It, too, is of general applicability and relatively easy to code, and is particularly well-suited for problems requiring a frequency response curve. However, the convergence rate for this method (for harmonic excitation) can be shown to be equivalent to the ADI Helmholtz solution technique employing a single spatially-variable but non-optimum acceleration parameter. It is therefore expected to converge much slower than the present twoparameter ADI method, and in fact this has been confirmed in comparison tests using several different test problems.

The capability of the present technique to model complex structures containing many resonant reflections is aptly illustrated by considering the grating out-coupler shown in Figure 2. Here a slightly detuned second-order grating is etched into the waveguide shown in order to outcouple the guided mode into the air region at the left in the figure. Radiation scattered into the substrate by the grating is reflected by a 10.5 period quarter-wave stack of GaAs/AlAs dielectric layers designed using a separate analysis to be $90 \%$ reflective. The 388 x 980 mesh chosen for this problem allowed the simulation of 40 grating periods with about 22 mesh points per wavelength in each direction inside the waveguide for good overall accuracy. The input waveform used was an approximate eigenmode of the waveguide as modified by the grating. The absorption regions were constructed using an index of refraction whose real part matched the adjoining material and whose imaginary part was linearly ramped from zero at the adjoining interface 
to a maximum value at the boundary chosen to minimize reflectivity. Typical reflectivities for the absorbers near the top and left boundaries were $<10^{-4}$. The right boundary absorber was considerably more reflective since it was positioned behind the dielectric stack. Convergence to relative accuracies of $10^{-4}$ was obtained in 750 iterations.

Intensity contours for the converged solution are shown (not to scale) in Figure 3. The far-field emission pattern for radiation into the air region was determined by Fourier transformation of the computed field just to the right of the absorber, and consisted primarily of a single lobe directed 9.9 degrees backwards from normal with a FWHM of 4.4 degrees. This compares well with the analytic values of 9.85 and 4.7 degrees, resp., with the latter figure being the estimate for a diffraction-limited source of length $16 \mu \mathrm{m}$. As reported previously ${ }^{10}$, the grating efficiency (defined as the fraction of beam energy radiated away per unit grating length) was found to be very sensitive to the position of the reflector stack, with a maximum corresponding to a reflector spacing of $0.8294 \mu \mathrm{m}$ from the right waveguide boundary. This maximum corresponds to in-phase addition of the reflected radiation with that radiated into the air directly from the grating. Other periodically-spaced maxima were observed, separated by a spacing of $0.1914 \mu \mathrm{m}$, in good agreement with the expected value of $0.1926 \mu \mathrm{m}$. This calculation demonstrates the capability of this technique for modeling structures characterized by resonant interference from multiple reflections in two perpendicular directions. It also allows the design of gratings with outcoupling efficiencies of nearly $250 \mathrm{~cm}^{-1}$ using carefully positioned reflectors and deep etching techniques. 
Although the calculation just described could have been performed using a different modeling technique (such as by a series expansion method $\left.{ }^{3}\right)$, the latter requires significant specialized knowledge of grating theory, and would be inapplicable to other problems. In contrast, the present solution required only that extra coding necessary to define the grating structure, and thus the Helmholtz solution technique described in this letter has very wide applicability. The calculation presented above utilized over 380,000 mesh points, yet required only 35 MBytes of storage, and was completed in less than two hours on an IBM R/S-6000 workstation. In contrast, direct solution methods are totally out of the question for problems of this size, and other iterative techniques are considerably more complicated to code. In addition, the method generalizes directly to $3 \mathrm{D}$ if the $\mathrm{ADI}$ algorithm is replaced by that due to Douglas', although storage and runtimes will increase linearly with the number of mesh points.

In summary, a previously well-known technique (ADI) has been rendered practical for solution of the Helmholtz Equation by the employment of semi-optimized Wachspress-like acceleration parameters, and successfully applied to model complex reflective structures of significance in photonics research. 


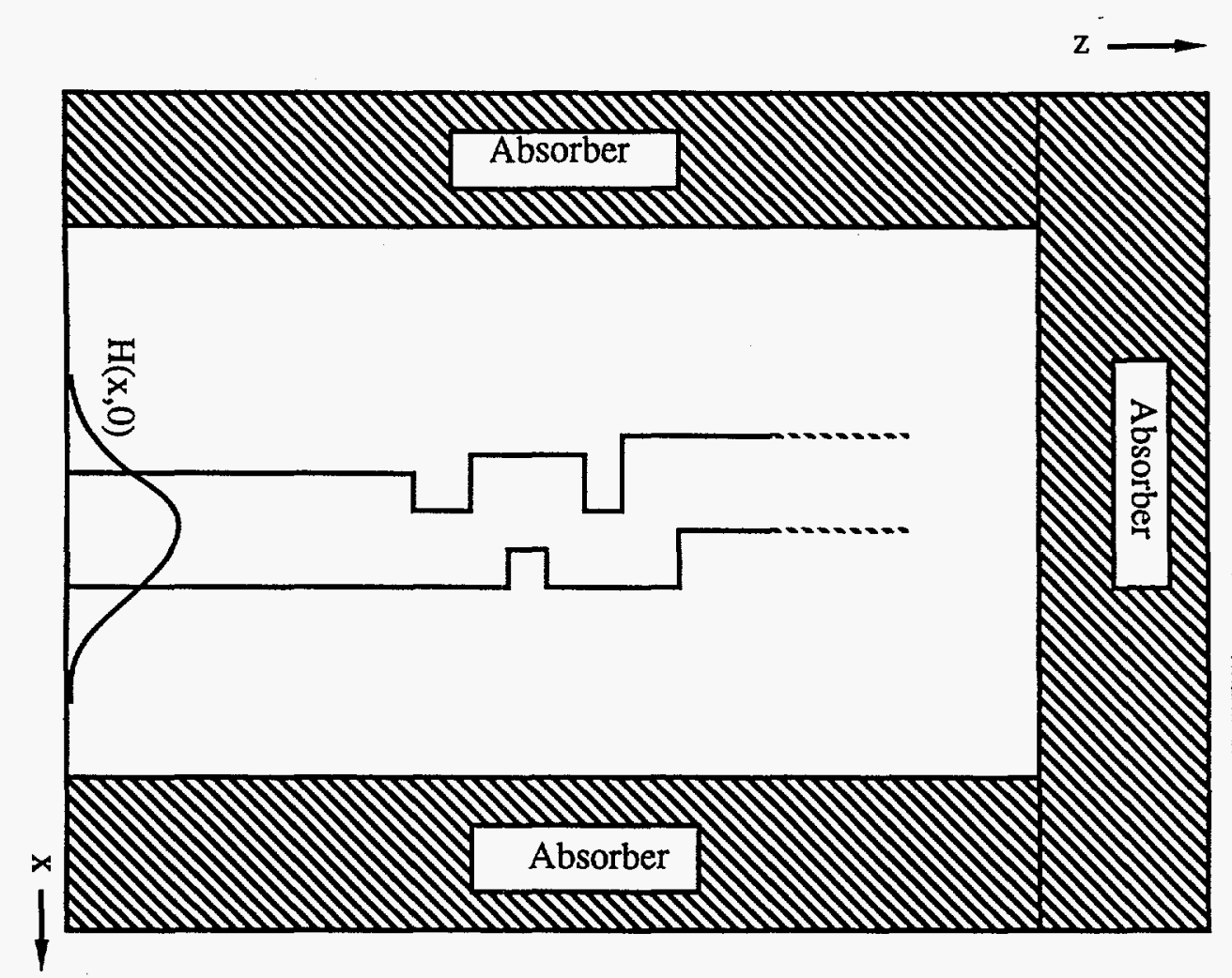

Fig.1 Schematic illustrating a typical problem geometry for the ADI Helmholtz solution technique described in this letter. An input (guided) mode enters at the lower boundary, scatters off the structure of interest, and is absorbed in the regions adjacent to the other three boundaries.

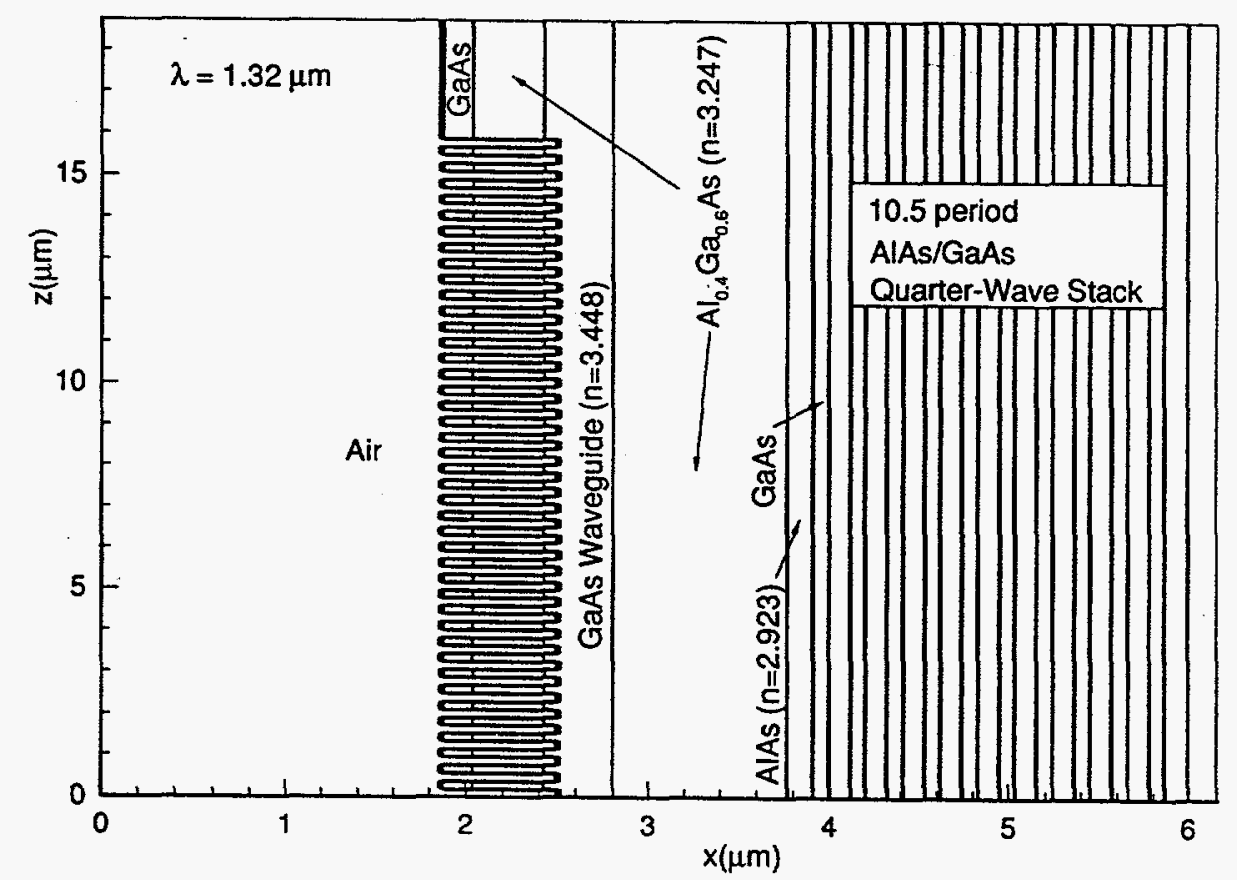

Fig.2 Geometry (not to scale) for the sample problem discussed in the text. A second-order grating has been etched into the waveguide as shown. Light scattered towards the substrate is reflected by the 10.5 period dielectric stack. 


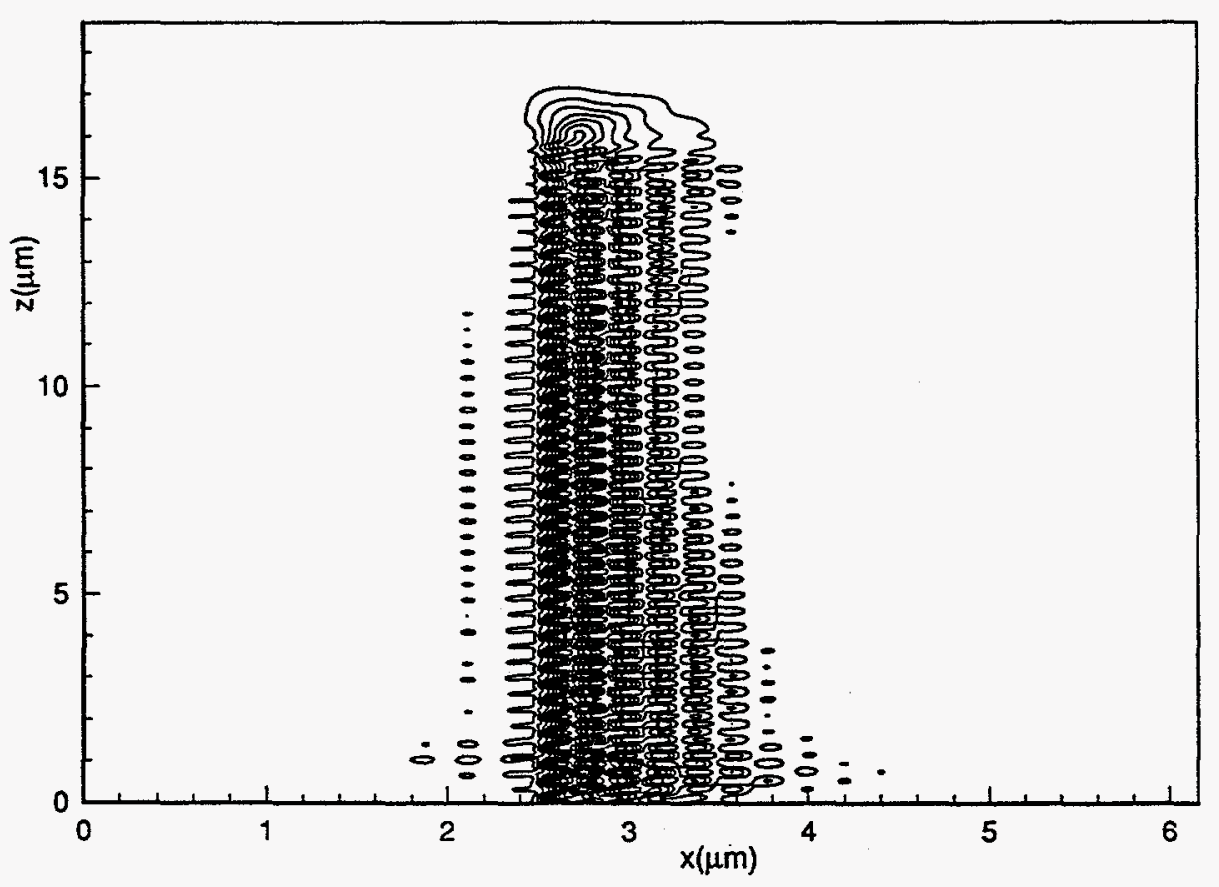

Fig.3 Intensity contours for the converged solution of the waveguidewith-grating problem shown in Fig. 2 and discussed in the text. The input guided mode was an approximate eigenmode of the waveguide as modified by the presence of the grating. 


\section{References}

1. P. Kaczmarski and P. Lagasse, Electron. Lett. Vol. 24, 675(1988).

2. C. H. Henry, IEEE J. Quantum Electron., Vol. 27, No. 3.523(1991).

3. D. Marcuse, Bell Sys. Tech. Journal, Vol. 55, No. 9,1295(1976).

4. A. Hardy, D. F. Welch, and W. Streifer, IEEE J. Quantum Electron. Vol.25, No. 10, $2096(1989)$.

5. E. I. Wachspress, J. Soc. Indust. Appl. Math., Vol. 10, No. 2, $339(1962)$.

6. A. Bayliss, C. I. Goldstein and E. Turkel, J. Comp. Phys. Vol. 49, $443(1983)$.

7. R. S. Varga, Matrix Iterative Analysis, (Prentice Hall, Inc., Englewood Cliffs, N.J., 1963), Chap. 7, esp. p.212.

8. E. L. Wachspress, Iterative Methods for Large Linear Systems, (Academic Press, 1990), Chap. 15.

9. W. P. Huang, S. T. Chu, A. Goss, and S. K. Chaudhuri, IEEE Phot. Tech. Lett. Vol. 3, No. 6, 524(1991).

10. D. Mehuys, A. Hardy, D. F. Welch, R. G. Waarts and R. Parke, IEEE Phot. Tech. Lett. Vol. 3, No. 4, 342(1991). 


\section{Appendix G. LDRD Summary}

Reports and publications resulting from the work: 6

G.R. Hadley, Numerical Simulation of Reflecting Structures via Solution of the 2D Helmholtz Equation, 1994 Integrated Photonics Research Meeting, Technical Digest Series Vol.3 p.42, THD1-1.

M.E. Warren, et al. 1994 Application of Diffractive Optics to Photonic Integrated Circuit Packaging, Diffractive Optics Vol. 111994 OSA Technical Digest Series.

S.H. Kravitz, et al. 1993. Waveguide-To-Fiber Coupling Using A Second-Order Grating And An Anamorphic Binary Optic, IEEE Lasers and Electro-Optics Society 1993 Annual Meeting.

S. H Kravitz ,et al. High-Performance GaAs/AllGaAs Optical Modulators: Their Performance and Packaging for Microwave Photonic Integrated Circuits, Asia Pacific Microwave Conference Workshop Proceedings Dec. 6-9, 1994 WS2-3 p. 45.

R.F. Carson, et al. 1993. Micro-Optic-Based Interconnect and Packaging for GaAs Photonic Integrated Circuits, Optical Society of America Annual Meeting October 1993, Toronto, Canada.

Pamela Seigal, et al. National Science Foundation sponsored, International Workshop on Optoelectronic Packaging Science, Santa Barbara, CA July 28-30, 1993, Poster "Silicon MCM Substrates for Integration of III-V Photonic Devices and CMOS IC's".

Number of patent disclosures: 2

Number of patent applications: 2

Number of Copyrights: None

Number of students: None

Number of post docs: None

Number of permanent technical or scientific staff: None

Number of awards (and their names): None

Number of new non-LDRD funded projects and their amounts: None 


\section{Distribution:}

1 MS0320 LDRD Office, 1010

1 MS1079 A. D. Romig, 1300

1 MS0603 J.Y.Tsao, 1311

1 MS0603 R. E. Smith, 1311

1 MS0603 A. Owyoung, 1312

1 MS0603 G.R. Hadley, 1312

1 MS0603 M. E. Warren, 1312

1 MS1077 H. T. Weaver, 1321

1 MS0603 T. M. Bauer, 1321-1

1 MS0603 T. E. Zipperian, 1322

1 MS0603 S. H. Kravitz, 1322

1 MS0603 J. R. Wendt, 1322

1 MS1082 D. W. Palmer, 1333

1 MS1082 P. K. Seigal, 1333

1 MS0874 G. V. Herrera, 1342

15 MS0874 M. G. Armendariz, 1342

1 MS9018 Central Technical Files, 8523-2

5 MS0899 Technical Library, 4414

1 MS0619 Print Media, 12615

2 MS0100 Document Processing, 7613-2

For DOE/OSTI 